\title{
High resolution numerical weather prediction over the Indian subcontinent
}

\author{
T S V ViJAYA KumaR ${ }^{1,2, *}$ and T N KRIShnAmuRTi ${ }^{1}$ \\ ${ }^{1}$ Department of Meteorology, Florida State University, Tallahassee, Fl 32306-4520, USA. \\ ${ }^{2}$ Present address: Environmental Modeling Center, National Centers for Environmental Prediction, Camp \\ Springs, Maryland, USA. \\ *e-mail: vijay.tallapragada@noaa.gov
}

\begin{abstract}
In this study, the Florida State University Global Spectral Model (FSUGSM), in association with a high-resolution nested regional spectral model (FSUNRSM), is used for short-range weather forecasts over the Indian domain. Three-day forecasts for each day of August 1998 were performed using different versions of the FSUGSM and FSUNRSM and were compared with the observed fields (analysis) obtained from the European Center for Medium Range Weather Forecasts (ECMWF). The impact of physical initialization (a procedure that assimilates observed rain rates into the model atmosphere through a set of reverse algorithms) on rainfall forecasts was examined in detail. A very high nowcasting skill for precipitation is obtained through the use of high-resolution physical initialization applied at the regional model level. Higher skills in wind and precipitation forecasts over the Indian summer monsoon region are achieved using this version of the regional model with physical initialization.

A relatively new concept, called the 'multimodel/multianalysis superensemble' is described in this paper and is applied for the wind and precipitation forecasts over the Indian subcontinent. Large improvement in forecast skills of wind at $850 \mathrm{hPa}$ level over the Indian subcontinent is shown possible through the use of the multimodel superensemble. The multianalysis superensemble approach that uses the latest satellite data from the Tropical Rainfall Measuring Mission (TRMM) and the Defense Meteorological Satellite Program (DMSP) has shown significant improvement in the skills of precipitation forecasts over the Indian monsoon region.
\end{abstract}

\section{Introduction}

Numerical weather prediction (NWP) is one of the challenging tasks in meteorology and has been subjected to extensive research in the last few decades. Current developments in NWP include the utilization of very high-resolution global and regional models, new methods for discretization of the dynamical equations, inclusion of more sophisticated physical processes, ensemble forecasting, and coupled ocean-atmosphere-land models. In addition, these models are used to understand the nonlinear dynamics of the atmosphere and the internal structure and evolution of storms (e.g., hurricanes, squall lines, mesoscale convective complexes, and snowstorms) in a better way. Progress in tropical weather prediction has been hindered for a long time by lack of data over the vast oceanic regions and inadequate understanding of the physical processes of weather patterns as compared to the extra-tropics.

The recent inclusion of space-borne remotely sensed data collection systems and application of new techniques in data assimilation provided an impetus to NWP studies, since it is possible to have a better description of the initial state of the atmosphere utilizing this data. Another major contribution for improving skills of the numerical

Keywords. Numerical weather prediction; spectral modeling; physical initialization; high resolution regional models; multimodel superensemble forecasts. 
model forecasts came from the use of ensemble forecasting methods, where different model forecasts are statistically combined to give a better prediction. In the last two decades, weather forecasts have become much more skillful and reliable. For example, in the mid-latitudes, today's 3-day forecasts are about as accurate as the one-day forecasts used to be twenty years ago. The improvement in skill over the last 40 years of NWP is due to increased power of supercomputers, allowing much finer horizontal resolution and fewer approximations in the operational atmospheric models; improved representation of small-scale physical processes (clouds, precipitation, turbulent transfers of heat, moisture, momentum and radiation) within the models; use of more accurate methods of data assimilation; increased availability of data, especially satellite and aircraft data over the oceans and the southern hemisphere; and sophisticated statistical ensemble methods that remove the model biases and improve the accuracy of the forecasts.

In a series of remarkable papers, Lorenz (1963, $1965,1968)$ made the fundamental discovery that even with perfect models and perfect observations, the chaotic nature of the atmosphere would impose a finite limit of about two weeks to the predictability of the weather. Because the skill of the forecasts decreases with time, Epstein (1969) and Leith (1974) suggested that instead of performing deterministic forecasts, stochastic forecasts providing an estimate of the skill of the prediction should be made. The only computationally feasible approach to achieve this goal is found to be through ensemble forecasting (Kalnay 2002).

Though tropical NWP has gained importance in recent times, the progress is slow; the forecasts over the monsoon domain are usually poor after about a 24-h period and sometimes unrealistic on day-2 and day-3. Particularly over the Indian domain, the forecasting skill of operational NWP models is quite inapt. Statistical methods of long range forecasts of monsoon rainfall dominated the numerical forecasts in India and officially the India Meteorological Department (IMD) uses a set of statistical models (Thapliyal and Kulshrestha 1992; Thapliyal 1997; Rajeevan et al 2004) for sub-seasonal to seasonal forecasts during the summer monsoon period from June to September. On short to medium range, the National Centre for Medium Range Weather Forecasting (NCMRWF) uses a spectral model at T80 resolution and issues operational real time forecasts. (A list of acronyms is provided in table 1.) IMD uses a Quasi Lagrangian Model (QLM) and a Limited Area Model (LAM) on a daily basis to provide numerical forecasts for the Indian region. However, the forecast skill of these models is very
Table 1. List of Acronyms.

\begin{tabular}{|c|c|}
\hline Acronym & Full form \\
\hline BMRC & Bureau of Meteorology Research Center \\
\hline $\mathrm{Cal} / \mathrm{Val}$ & Calibration/Validation \\
\hline $\mathrm{CMC}$ & Canadian Meteorological Center \\
\hline DMSP & Defense Meteorological Satellite Program \\
\hline DSS & Data Storage Section \\
\hline ECMWF & $\begin{array}{l}\text { European Centre for Medium Range } \\
\text { Weather Forecasts }\end{array}$ \\
\hline FSUGSM & $\begin{array}{l}\text { Florida State University Global } \\
\text { Spectral Model }\end{array}$ \\
\hline FSUNRSM & $\begin{array}{l}\text { Florida State University Nested Regional } \\
\text { Spectral Model }\end{array}$ \\
\hline GPROF & Goddard Profiling (algorithm) \\
\hline $\mathrm{IMD}$ & India Meteorological Department \\
\hline JMA & Japan Meteorological Agency \\
\hline NASA & $\begin{array}{l}\text { National Aeronautics and } \\
\text { Space Administration }\end{array}$ \\
\hline NCAR & $\begin{array}{l}\text { National Centers for } \\
\text { Atmospheric Research }\end{array}$ \\
\hline NCEP & $\begin{array}{l}\text { National Centers for Environmental } \\
\text { Prediction }\end{array}$ \\
\hline NCMRWF & $\begin{array}{l}\text { National Centre for Medium Range } \\
\text { Weather Forecasting }\end{array}$ \\
\hline NESDIS & $\begin{array}{l}\text { National Environmental Satellite, Data, } \\
\text { and Information System }\end{array}$ \\
\hline NOAA & $\begin{array}{l}\text { National Oceanic and Atmospheric } \\
\text { Administration }\end{array}$ \\
\hline NRL & Naval Research Laboratory \\
\hline NWP & Numerical Weather Prediction \\
\hline OLR & Outgoing Longwave Radiation \\
\hline PI & Physical Initialization \\
\hline RMSE & Root Mean Square Error \\
\hline $\mathrm{RPN}$ & Recherche Prévision Numérique \\
\hline SST & Sea Surface Temperature \\
\hline $\mathrm{SSM} / \mathrm{I}$ & Special Sensor Microwave/Imager \\
\hline TMI & TRMM Microwave Imager \\
\hline TRMM & Tropical Rainfall Measuring Mission \\
\hline UKMO & United Kingdom Meteorological Office \\
\hline UTC & Universal Time Constant \\
\hline
\end{tabular}

less, particularly for important variables like rainfall (Roy Bhowmik et al 2001; Rama Rao et al 2005). Hence, there is a dire necessity for efforts to improve performance of the numerical models in short-range NWP on a real-time basis for the Indian region. The FSU global and regional models FSUGSM and FSUNRSM have shown significant performance in simulating the features of the global tropics, especially of Indian summer monsoon and tropical cyclones (Krishnamurti et al 1984, 1995, 1998; Cocke 1998; Williford et al 1998, etc.). Having observed the merits of these two models, this paper aims to examine the skills of these models in a detailed way and explore the possibility of using these models for efficient weather forecasts over the Indian sub-continent. The motivation for 
this study came from the fact that the forecasting skills of FSUGSM and FSUNRSM improved with increase in the model horizontal resolution, optimization of model parameters and incorporation of physical initialization of observed rain rates.

This study also includes forecasts from multimodels of a number of global operational centers that are combined through a sophisticated statistical tool known as the 'superensemble', developed by Krishnamurti et al (1999, 2000a, 2000b, 2001). This method differs from the conventional ensemble mean where all member model forecasts are given equal weight. Superensemble collectively removes the bias of individual member models at every grid point, at every vertical level and at each time of forecast. Different weights are assigned to each member model at every grid point for each variable based on the model's past performance examined in a training phase of the superensemble. A multianalysis superensemble component is included in this study, which is based on the FSUGSM that utilizes TRMM and SSM/I data sets and a number of rain rate algorithms. The difference in the analysis arises from the use of these rain rates within physical initialization that produces distinct differences among these components in the divergence, heating, moisture, and rain rate descriptions. A total of 12 models, of which 7 represent global operational models and 5 represent multianalysis forecasts from FSU model initialized by different rain rate algorithms, are included in the multianalysis/multimodel superensemble system studied here.

There were three major goals of this study. The first of these was to show that the forecasts made therefrom using a regional spectral model carry a higher skill compared to global models at comparatively lower resolution. The second was to obtain a very high nowcasting skill of rainfall using a highresolution regional spectral model, while the third goal was to demonstrate the performance of the superensemble methodology for short-range wind and precipitation forecasts.

The salient features of the FSUGSM and FSUNRSM are given in section 2. The experimental setup and some important results from these models are presented in section 3. Section 4 deals with the high resolution physical initialization within the FSUNRSM along with explicit specification of soil temperatures. Section 5 describes the data, experimental setup and results obtained using the multimodel/multianalysis superensemble method, and a detailed summary, conclusions and scope for future work are provided in section 6 .

\section{FSU global and regional spectral models}

\subsection{Florida State University Global Spectral Model}

The Florida State University Global Spectral Model (FSUGSM) is one of the state-of-the-art NWP models with special emphasis on tropical weather prediction. This model owes its origin to the Canadian spectral model RPN, Daley et al (1976), and has been used in various configurations to study a wide range of atmospheric phenomena. Typical configuration changes, such as spatial resolution, choice of parameterization schemes, length of model integration, etc. involve simple modifications to initial model parameters without changing the model code. A detailed documentation of the FSUGSM was given in Krishnamurti et al (1991) and Kumar (2000).

The resolution of the FSUGSM, both in the horizontal and vertical, can be varied to accommodate various research purposes. The model integration utilizes semi-implicit time differencing scheme with Asselin (1972) time filter. The vertical differential terms in the model equations are solved by applying finite-difference methods, while the horizontal differential terms are determined spectrally. All variables in the vertical are solved by using a centered differencing approach, except humidity which is handled by an upstream differencing scheme. The FSUGSM uses a standard approach for atmospheric spectral models, where linear terms in time tendencies are computed in the spectral space and non-linear terms are computed in the physical space on a Guassian grid (or transform grid). The FSUGSM has a wide range of physical processes to choose for specific objectives of research. Salient features of the FSUGSM are given in Appendix 1.

\subsection{Florida State University nested regional spectral model}

On account of their definite advantages over the grid point models, global spectral models are being used by most of the operational NWP centers for short and medium range weather forecasts. The accuracy of the numerical forecasts by these models (both spectral and grid-point) increases as the model resolution is increased, since at a higher resolution, these models are able to capture finer scales that are necessary to define and forecast smaller, regional scale weather systems. For a very high-resolution forecast over some specified limited area over the globe, using a very high-resolution limited area model in conjunction with a relatively 
low-resolution global model is computationally more advantageous than increasing the resolution of the global model. Until recently, most of the limited area models used grid-point or finite difference methods. However, these models exhibit problems such as phase and biased errors and non-linear instability (Roache 1976; Baer 2000). Computing space derivatives with high-order accuracy also does not resolve these problems. Besides, economical time integration methods, such as semi-implicit schemes, are not very convenient to implement in a grid-point model. To overcome these problems and exploit the advantages of spectral methods, a number of limited area spectral models have been developed for operational use at NCEP and ECMWF (Tatsumi 1986; Hoyer 1987; Juang and Kanamitsu 1994).

The nested regional spectral model developed at FSU (FSUNRSM) was first used in Atlantic hurricane prediction studies (Cocke 1998) and has undergone considerable enhancement since then. FSUNRSM uses a perturbation method similar to that used at NCEP and ECMWF but largely differs from these models, particularly in the areas of map projection, boundary relaxation procedure, finite differencing schemes, etc. The FSUNRSM was designed to be compatible with the FSUGSM. These models have the same vertical $\sigma$ coordinate system, horizontal diffusion, Asselin time filter, semi-implicit time integration and physical parameterization schemes. The regional model solution is the sum of the global model solution plus the perturbations. These perturbations are spectrally represented by bi-periodic trigonometric series and are relaxed at the lateral boundaries so that the regional fields approach the global model solution. Only the perturbations are represented by the trigonometric functions, not the regional fields themselves. This enables a natural means to incorporate the lateral boundary condition. Depending on the variable, the perturbations satisfy a zero or mirror boundary condition. One advantage of the perturbation technique is that the global model predicts the large-scale flow whereas the finer-to-intermediate scales are predicted by the regional model. In most other regional models, the large scale flow is passed into the regional domain through a narrow blending or relaxation zone, typically a few grid points in width, at the lateral boundaries. The perturbation method incorporates the large-scale flow throughout the regional domain.

The global model is run first, and the global output fields are then spectrally transformed to the regional grid at every 3 hours. The use of a spectral transformation eliminates the need for horizontal interpolation, thus reducing error. These transformed global fields are then linearly interpolated in time to the time steps of the regional model. The perturbation time tendencies at initial time of the regional model integration are set to zero. At each time step, the perturbations are added to the global fields to obtain the full regional fields, and the nonlinear dynamical and physical tendencies are computed. The perturbation time tendencies are obtained by subtracting the regional time tendencies from the global time tendencies (which were obtained by an inverse semi-implicit algorithm). Currently, only one-way nesting is done; the regional solution does not feed back into the global model.

The FSUNRSM uses a Mercator projection in the horizontal. The advantage of using the Mercator type projection lies in the fact that it incorporates a means of compatibility between the FSUGSM and the FSUNRSM, thus allowing both the global and the regional models to use the same longitudinal co-ordinate. This convenience results in a reduction of computational time, as the global variables and derivatives can be easily transformed to the regional grid via a fast Fourier-Legendre transform. FSUNRSM has slip wall lateral boundary conditions with respect to perturbations, i.e., there can be perturbation wind flow along the boundary but not across the boundary. Accordingly, no advection of perturbations of scalar fields like temperature, pressure and moisture is allowed across the boundaries. The mathematical formulation and detailed description of the FSUNRSM are given in Kumar (2000).

\subsection{Physical initialization}

Physical initialization (Krishnamurti et al 1984, 1991 ) is a powerful tool, which primarily assimilates satellite derived observed rainfall distributions along with calculated surface fluxes of moisture to produce a physically consistent and more realistic spin-up of the initial state. This is accomplished through a number of reverse physical algorithms within the assimilation mode. Physical initialization is invoked $24 \mathrm{~h}$ prior to the initial forecast start time. By using a Newtonian relaxation process, certain forecast variables are relaxed until they converge to that of the observed, satellite derived or computed known variables. Thus the initial fields of mass divergence, moisture sink, surface pressure, vertical distribution of the humidity variable and surface fluxes of moisture as proposed by the model are modified to be in agreement with the observed rain rates, surface fluxes and the OLR. The observed rain rates are derived from DMSP SSM/I datasets. These datasets have a lot of gaps in the global coverage of 
rainfall distribution, and are hence filled with the rain rates obtained from the geo-stationary satellite based OLR fields. The total field is a merged SSM/I - OLR product. The process of physical initialization incorporates a reverse cumulus parameterization scheme, a reverse similarity algorithm, an OLR matching algorithm and Newtonian relaxation of the model variables. The brief procedure of the PI is as follows:

Step 1: Diagnostic calculations of the surface fluxes, and humidity analysis consistent with the surface fluxes, rainfall rates and the net OLR. This step is done through a set of reverse physical algorithms. The first part uses a reverse similarity theory to compute the surface evaporative fluxes from the sum of the apparent moisture sink $\left(\mathrm{Q}_{2}\right.$, Yanai et al 1973) and the observed rain rates. The surface sensible heat fluxes are obtained from the knowledge of the apparent heat source $\left(Q_{1}\right.$, Yanai et al 1973) and the net radiative heating. After the assimilation procedure, the resulting surface fluxes tend to exhibit consistency with the observed rain rates. In order to solve for the potential temperature and the moisture variable (assumed to be unknowns) at the top of the constant flux layer, the Yanai fluxes of sensible heat and latent heat are used as input to the similarity theory (Businger et al 1971). The second part is obtained through a reverse cumulus parameterization algorithm which reanalyzes the vertical distribution of specific humidity such that the rainfall implied by the cumulus parameterization algorithm (modified Kuo scheme, Krishnamurti et al 1983) matches the given observed rainfall rates. Since the observed rain rates are known quantities, the specific humidity in the vertical can be obtained through an iterative procedure. The humidity measurements from conventional radiosonde above $500 \mathrm{hPa}$ surface are generally quite unreliable and hence the humidity distribution is restructured using an OLR matching algorithm between the model calculated OLR and the satellite based observations through a local structure function for the moisture variable. More details on the computational aspects of these reverse algorithms are described in Kumar (2000), Krishnamurti et al (1991) and Shin (2001).

Step 2: Incorporation of these computed fluxes, observed (estimated) rain rates and observed clouds during the pre-integration phase, where the diagnostically computed fluxes (from Yanai's formulation) (first part of step 1), 'observed' rain rates and 'observed' clouds (through OLR matching algorithm) are incorporated into the preintegration phase of the model, wherein an additional term is added to the model's dynamical and thermodynamical equations. The additional term defines a Newtonian Nudging that relaxes model-forecast values to certain observed estimates. This is the crucial step in the physical initialization procedure. Here the vorticity, divergence and pressure tendency equations are subjected to the relaxation, where the spectral equations take the form

$$
\frac{\partial A_{l}^{m}}{\partial t}=F_{l}^{m}(A, t)+N(A, t) \bullet\left(A_{l}^{0 m}-A_{l}^{m}\right) .
$$

Here $N$ represents the relaxation coefficient, $A_{l}^{0 m}$ a specified future value to which the Newtonian relaxation is aimed at, and $F_{l}^{m}(A, t)$ the forcing term of the equations of variable $A$. (l, $m$ are zonal and meridional wave numbers.) Solving the above equation iteratively, the variable $A_{l}^{m}$ attains a solution that falls between the model derived value and the observed value during this relaxation. If the relaxation coefficient $(N)$ is too large, the model state will not be in primitive equation balance, and if $N$ is too small, there will be little impact on the evolution of the model state during this assimilation. The relaxation coefficients employed in our study are $1 \times 10^{-4} \mathrm{~s}^{-1}$ for vorticity and surface pressure, and $5 \times 10^{-5} \mathrm{~s}^{-1}$ for divergence. There is no relaxation for humidity variable; it is reanalyzed using the reverse algorithms and OLR matching.

\section{NWP experiments from FSUGSM and FSUNRSM}

The capability of FSUGSM and FSUNRSM in predicting the monsoon weather over the Indian subcontinent for the month of August 1998 is examined in detail in this section. The FSUGSM was run with two different horizontal resolutions, T42 and T126, corresponding to approximately 2.8 degrees and 0.94 degrees latitude/longitude (Guassian grid) near the equator respectively. The vertical resolution is fixed for both T42 and T126 at 11 levels for the moisture variables and 14 levels for the remaining variables. These levels are unevenly spaced (staggered) between 10 and $1000 \mathrm{hPa}$. The model is capable of increased vertical resolution to as many as 29 levels. The current version of FSU model is run at T126L29, but requires more time (almost double) to complete a 3-day forecast. The results, when interpolated to the standard pressure levels, did not show any marked differences. Keeping in view of the computational resources and operational feasibility, it was thought initially to use 14 levels in the vertical and two different horizontal resolutions for the global model (T42 and T126). Three-day global forecasts were made for each day of August and the output was stored at every 6 -h interval. The initial 
data sets and surface parameters had a resolution of approximately 1.125 degrees (T106) and were taken from the ECMWF operational analysis at 1200 GMT of every day. These data sets were interpolated to the respective global model resolutions and were used to initialize the models. These same data sets were also used subsequently for the forecast verification. The initial spectral start files for each of these model runs consist of all basic variables $(u, v, w, z, T$ and $q$ ) for all 14 levels (except for moisture where only 11 levels were used). The surface parameters like SST, orography, ice cover, land category and other boundary conditions were acquired from the NCAR DSS and were transformed to the Gaussian grid of required resolution. The SSTs were fixed at the initial start time of any 3-day forecast. The topography was specified using US Navy's high-resolution orographic data sets. The FSUNRSM was also run at two different horizontal resolutions - 1 degree and half-degree. While the regional fields at 1 degree resolution were interpolated from the T42 experiments, regional fields at the half-degree resolution were taken from the T126 experiments.

The geographical domain covered by the regional model is approximately the same as the domain of study, i.e., $0-30^{\circ} \mathrm{N}, 60-120^{\circ} \mathrm{E}$. For computational feasibility in spectral space, currently the following conditions are necessary to set the regional domain: $2 \times($ nlonr -1$)$ must be equal to $2^{p} \times$ $3^{q} \times 5^{r}, p>0, q \geq 0, r \geq 0$; same is true for nlatr. nlonr and nlatr correspond to the number of longitude points and latitude points respectively, $p, q, r$ are integers. Also, the regional domain cannot cross the Greenwich meridian. To satisfy the above conditions, regional model for 1-degree resolution contains $65 \times 33$ points in the east-west and northsouth directions, with a starting longitude of $60^{\circ} \mathrm{E}$ and equator as the starting latitude, extending the domain to $125^{\circ} \mathrm{E}$ and $33^{\circ} \mathrm{N}$. The half-degree resolution is represented by 129 points in the eastwest and 65 points in the north-south with the same starting longitude and latitude. For presenting the results, we have chosen a uniform area comprising the region between equator and $30^{\circ} \mathrm{N}$ and $60^{\circ} \mathrm{E}-120^{\circ} \mathrm{E}$.

The prognostic output fields emanated from these model experiments are sea level pressure, accumulated precipitation, relative humidity, zonal and meridional components of wind, temperature, dew point temperature and geopotential height. The model output was interpolated to standard pressure levels (from sigma to $p$ ) using sophisticated vertical interpolation programs. Skills of the forecasts were measured for different parameters using RMS errors and spatial correlation coefficients. All the output fields from T42 experiments
Table 2. Selected list of experiments using different versions of FSU Global Spectral Model and FSU Nested Regional Spectral Model.

\begin{tabular}{|c|c|}
\hline Experiment & Description \\
\hline GT42 & $\begin{array}{l}\text { FSUGSM at a resolution T42 } \\
\text { (2.8125 degrees latitude/longitude on } \\
\text { Gaussian grid) in the horizontal with } \\
\text { default model parameters. }\end{array}$ \\
\hline GT126 & $\begin{array}{l}\text { FSUGSM at a resolution T126 } \\
\text { ( } 0.9375 \text { degrees latitude/longitude on } \\
\text { Gaussian grid) in the horizontal with } \\
\text { default model parameters. }\end{array}$ \\
\hline GT126PI & GT126 with physical initialization. \\
\hline R1T42PI & $\begin{array}{l}\text { Same as R1T42 but initial base fields are } \\
\text { taken from the output of a separate } \\
\text { GT42NEW experiment using physical } \\
\text { initialization from day- } 1 \text { to day } 0 \text {. }\end{array}$ \\
\hline R5T126PI & $\begin{array}{l}\text { Same as R5T126 but initial base } \\
\text { fields are taken from GT126PI. }\end{array}$ \\
\hline PIR5T126PI & $\begin{array}{l}\text { A separate experiment with R5T126PI } \\
\text { where physical initialization is applied } \\
\text { directly at the regional model level. }\end{array}$ \\
\hline
\end{tabular}

were linearly interpolated to a 1-degree lat./lon. resolution while output fields from T126 experiments were brought on to a uniform half-degree resolution. Based on the relative performance of each of the experiments conducted, only a total of five sets of experiments (from both low and high resolution categories) were chosen among the global and regional models and results from these experiments are presented in this section. Details of these selected experiments are provided in table 2 . The PI experiments use the physical initialization procedure illustrated in section 2.3 .

\subsection{The summer monsoon during August 1998}

The region of southeast Asia, particularly the Indian subcontinent and its adjoining oceanic region, experiences a characteristic seasonality of winds and precipitation, termed as the monsoon. The sequence of events that trigger the monsoon circulation are formation of heat low over northwest India preceding the transport of moisture into the land mass convergence of heat, profound cumulus convection and enhancement of diabatic heating and precipitation events. The onset of summer monsoon takes place towards the end of May and the monsoon prevails till the end of September. During 1998, the southwest monsoon set in over Kerala and south Tamilnadu on 2 June. After advancement towards most parts of the Indian subcontinent, the monsoon experienced a break situation during July 14-26 and regained its strength afterwards. The axis of the monsoon trough was north of its normal position on most of the days of August 1998. The monsoon was active or vigorous 

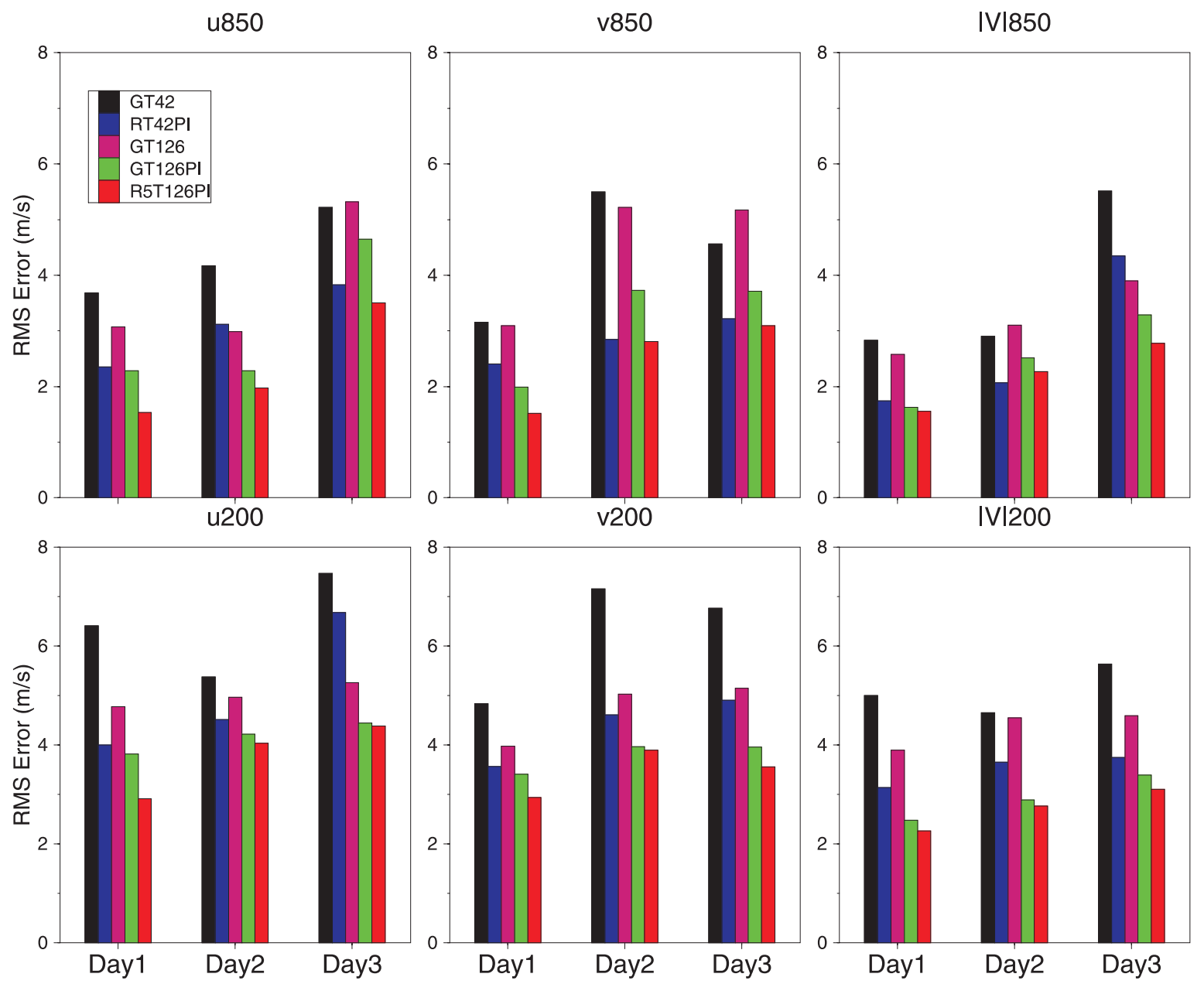

Figure 1. Mean RMS errors of zonal wind $(u)$, meridional wind $(v)$ and total magnitude of wind $|V|\left(\mathrm{m} \mathrm{s}^{-1}\right)$ at $850 \mathrm{hPa}($ top panels) and $200 \mathrm{hPa}$ (bottom panels) over the Indian monsoon domain for the month of August 1998. Details of experiments listed in the legend are provided in table 2.

in peninsular India during this month, with rainfall exceeding the normal in 13 meteorological subdivisions while 15 subdivisions received normal rainfall and the remaining 7 subdivisions experienced deficit rainfall (IMD, 1999). The pressure anomalies were positive over most parts of the country. Two weak low-pressure systems formed during this month, it is being one among very few occasions in August when no depression formed over the Indian seas throughout the month.

\subsection{Wind forecasts}

The mean RMS error of the zonal, meridional and total wind at $850 \mathrm{hPa}$ and $200 \mathrm{hPa}$ is presented in figure 1. This diagram gives an indication of the superior performance of the high resolution regional model (R5T126PI) over all other versions of the model. The global model with PI (GT126PI) also has shown better skill but it is the regional model that outperformed all other models, by having least RMS error for all components of the wind for both $850 \mathrm{hPa}$ and $200 \mathrm{hPa}$ levels. It is also evident from these histograms that the high-resolution numerical experiments using PI have shown major improvements over the lowresolution global/regional models. The impact of resolution on improving the wind forecasts is better seen in this figure where the mean RMS errors of wind forecasts from R5T126PI (high resolution regional model) are much lower than the GT42 (low resolution global model). This is not surprising, since increase in resolution in general, enhances the forecast skill. However, compared to the GT126 model outputs at approximately 1 degree resolution, results from the regional model experiments at 1 degree resolution (RT42PI) have shown superior performance, indicating the advantage of the use of regional models over the global models, apart from the impact of physical initialization. Though the order of magnitude of error was increased with the increase in the forecast period, the errors from the regional model were much less. About $40 \%$ of improvement was achieved using high-resolution regional model for 

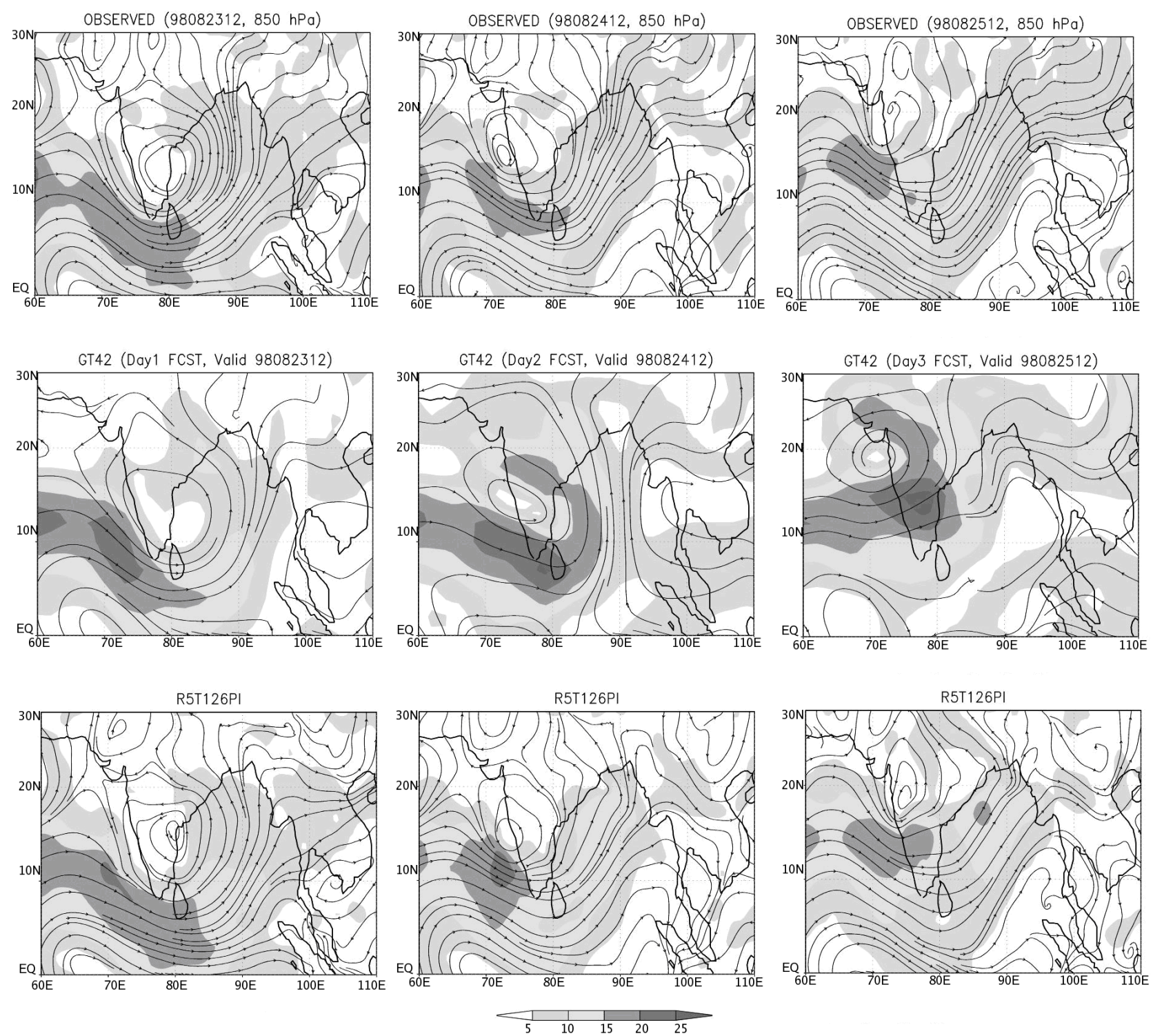

Figure 2. Streamlines and isotachs at $850 \mathrm{hPa}$ for 1, 2 and 3-day forecasts from GT42 and R5T126PI, valid on August 7, 8 and 9,1998 . Observed fields (ECMWF analysis) for corresponding days are shown in the top panel. Wind speeds (m s ${ }^{-1}$ ) are shown shaded. (X-axis from $60^{\circ} \mathrm{E}$ to $110^{\circ} \mathrm{E}$, Y-axis from equator to $30^{\circ} \mathrm{N}$.)

zonal wind forecasts on day- 1 , and about $25 \%$ on day-3. Approximately $30 \%$ improvement was noticed in the skill for wind forecasts at $850 \mathrm{hPa}$. More or less similar improvement was noticed for $200 \mathrm{hPa}$ wind forecasts as well.

The streamlines and isotachs at both 850 and $200 \mathrm{hPa}$ levels on a typical monsoon day are presented in figures 2 and 3 respectively, for observations, GT42 and R5T126PI. In these diagrams a sequence of three-day forecast starting from 23 to 25 August 1998 is presented. Shaded region represents the magnitude of the wind speed in $\mathrm{ms}^{-1}$. The monsoon circulation at $850 \mathrm{hPa}$, characterized by strong Somali jet near $10^{\circ} \mathrm{N}$ and $65^{\circ} \mathrm{E}$, the southwesterly flow across the Indian subcontinent, an elongated monsoon trough along the Indo-Gangetic region, a trough along the east coast of India and typical anticyclonic flow over the Arabian Sea were well represented by the R5T126PI experiment in its day-1 forecast as compared to other experiments. The control forecasts from GT42 (and even GT126, not shown here) without physical initialization could not capture any of these features. The wind speeds were better predicted by the R5T126PI. GT126PI also had shown some similarity with observed features (not shown here) but the R5T126PI outperformed its global counterpart. Similar improvement by R5T126PI could be observed even in day-2 and day-3 forecasts. By day-3, forecasts from 

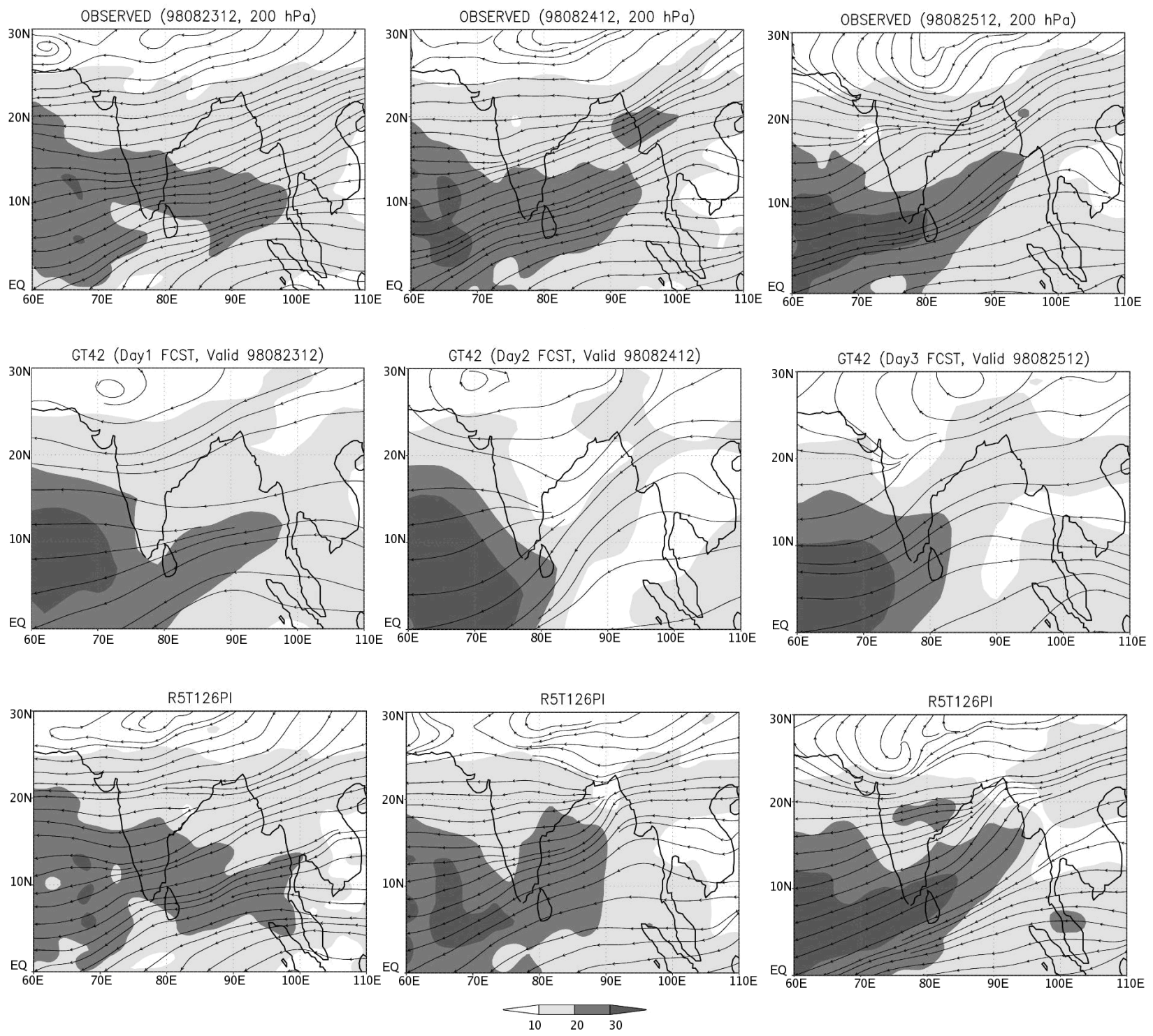

Figure 3. Same as figure 2, but for $200 \mathrm{hPa}$ streamlines and isotachs.

all other experiments including regional model at 1-degree resolution largely degraded except the R5T126PI. The $200 \mathrm{hPa}$ wind forecasts demonstrated in figure 3 also reveal similar improvements by R5T126PI compared to other model experiments. The Tibetan High, strong anticyclonic circulation over northern India and strong easterly jet stream were well captured by R5T126PI compared to other versions of the model. However, since at the time of designing the domain for the regional model, only the Indian landmass was considered keeping in of the computational restrictions and domain size parameters (nlonr and nlatr), and entire Tibetan plateau was not covered by the regional model domain. Even on day3 , the forecasts given by R5T126PI were strikingly close to the observed, indicating the fact that the regional models have better short-range prediction skills compared to global models. These results clearly demonstrate the improvement in forecast skill obtained by increased resolution as well as the impact of physical initialization.

\subsection{Precipitation forecasts}

Though practically it is not possible to predict precipitation rates with high accuracy, any improvement in its prediction is considered a significant achievement, particularly for the Indian region where its economy depends on the rainfall activity and its predictability. In figure 4 , the rainfall predictive skill over the Indian subcontinent is shown through the correlation coefficients. The three panels show correlation coefficients for forecast days 

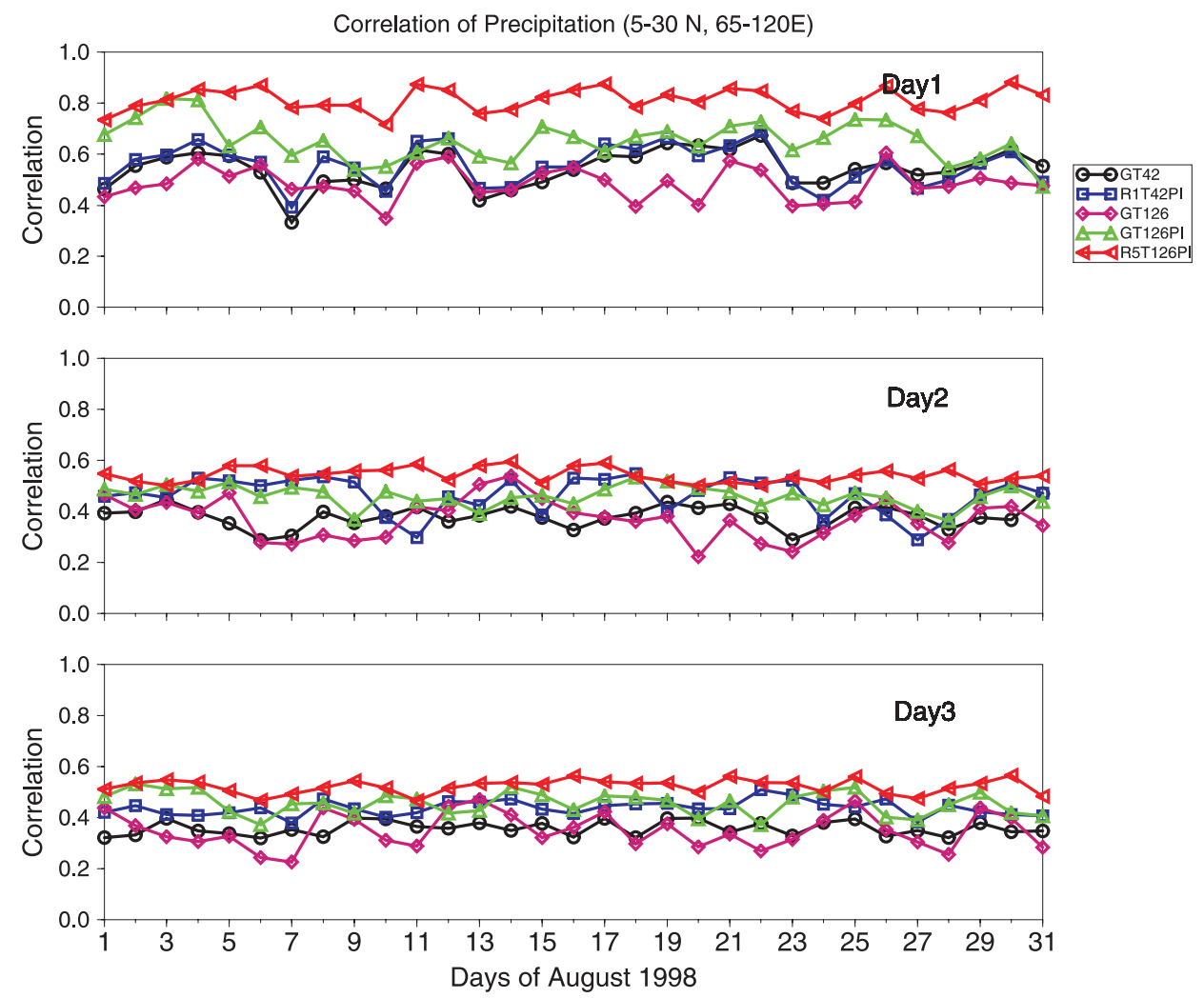

Figure 4. Time series of absolute correlation of observed and predicted rainfall over the Indian domain for the month of August 1998. Details of experiments listed in the legend are provided in table 2.
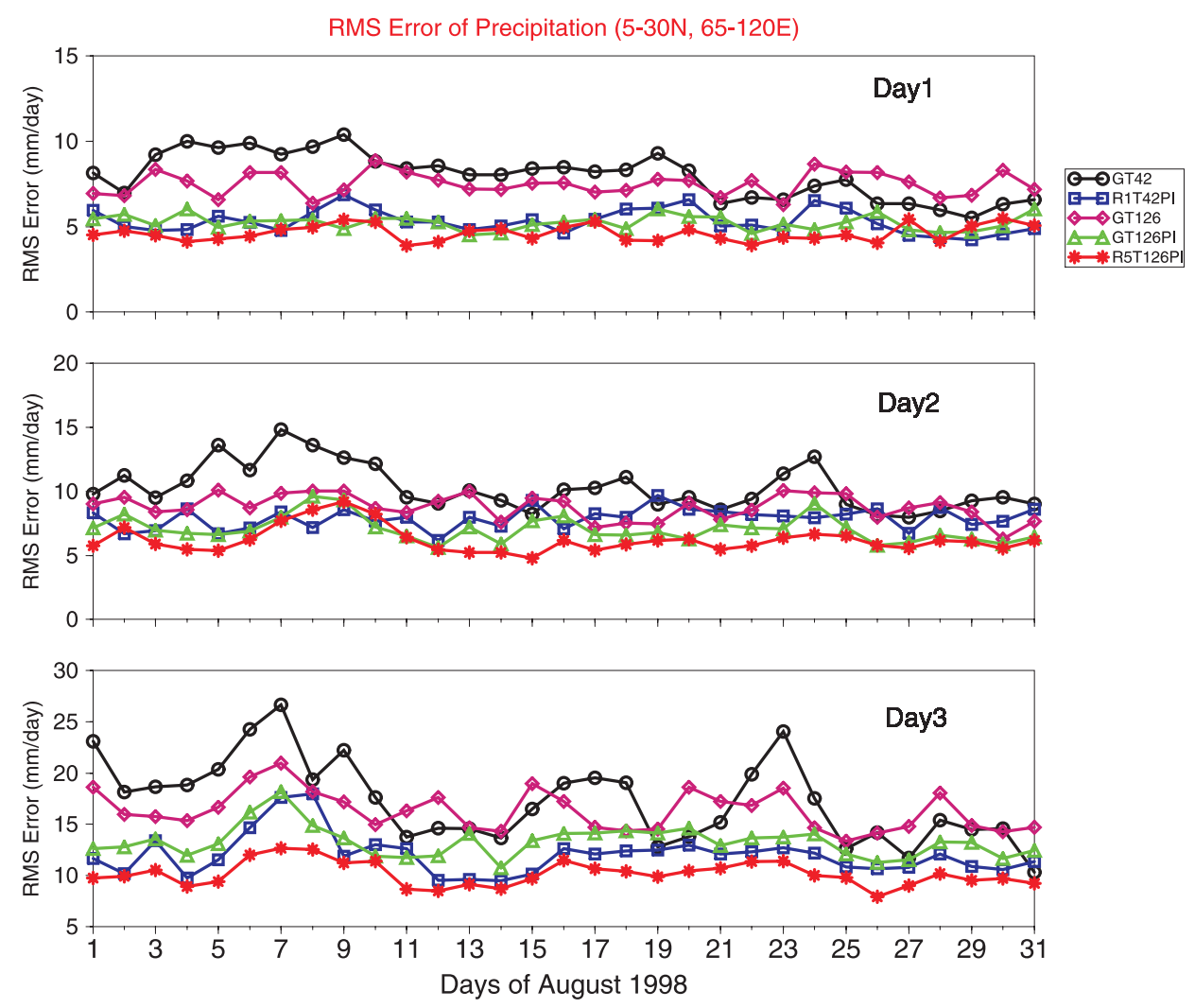

Figure 5. Same as figure 4 except for RMS error of precipitation in mm.day ${ }^{-1}$. 


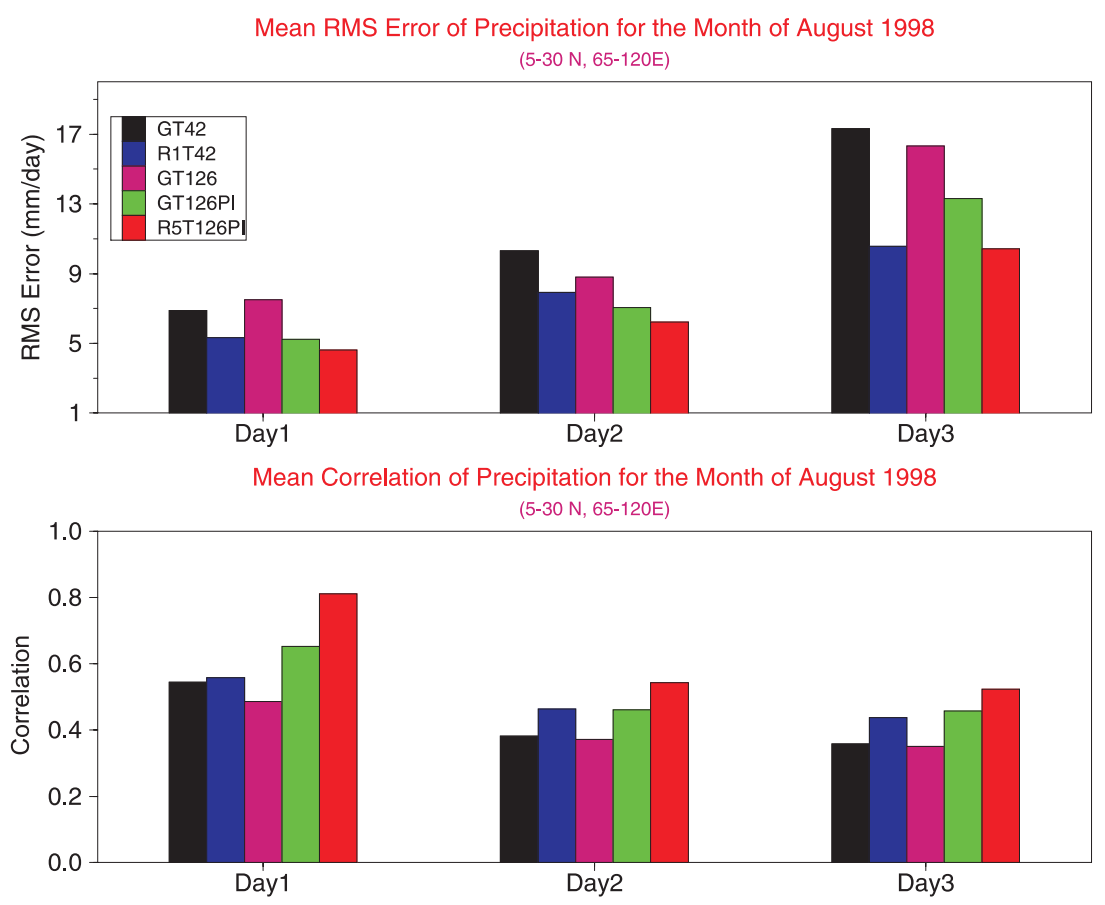

Figure 6. Mean RMS error $\left(\mathrm{mm} \cdot \mathrm{day}^{-1}\right)$ and mean correlation of precipitation for August 1998. Details of experiments listed in the legend are provided in table 2 .

1, 2 and 3. The line with the symbol '*' (that stayed almost on the top of all other lines in each panel) corresponds to the results from R5T126PI. The correlation coefficients for GT126PI are shown through the line with ' $\diamond$ ' symbol. These results show a marked skill at day-1 for the physically initialized experiments, which were substantially higher than the skill from the control runs. The skill was measured through the absolute correlation of the 24-h rainfall totals at the transformed grid squares between $5-30^{\circ} \mathrm{N}$ and $65-120^{\circ} \mathrm{E}$. R5T126PI had shown significant skill throughout the period of study on all days of forecast. The time series of RMS errors of rain rates is shown in figure 6. Here also the R5T126PI had least errors, followed by GT126PI. The superior performance of R5T126PI is a result of both high resolution (0.5 degree lat./lon.) and utilization of physical initialization. On day-1 forecast, R5T126PI had an RMS error of about $5 \mathrm{~mm} /$ day, which was increased to about $7 \mathrm{~mm} /$ day on day 2 and to about $12 \mathrm{~mm} /$ day on day-3 while the control experiment went to the other extremes, starting from about $12 \mathrm{~mm}$ /day of RMS error on day-1 to about $25 \mathrm{~mm} /$ day on day-3, which has no practical utility. The improvement in precipitation forecast skills by the regional model with physical initialization is a major result achieved in this study. The mean RMS errors and correlation coefficients of precipitation forecasts from all experiments are summarized in figure 6 . The R5T126PI outperformed all other models at each forecast range. A very high correlation of about 0.8 on day- 1 forecast was achieved through these experiments.

A sequence of 3-day forecast of 24-h accumulated precipitation $\left(\mathrm{mm} \cdot \mathrm{day}^{-1}\right)$ over the Indian monsoon domain is demonstrated in figure $7(\mathrm{a}, \mathrm{b}$, c). These figures show the rainfall forecasts valid on August 7, 8 and 9, 1998. The corresponding observed rainfall obtained from SSM/I+ OLR data sets is shown in the top left panel of each diagram. The high spatial correlation of rainfall predicted by R5T126PI (figure 6) is evident in these diagrams as compared to the low-resolution global model (GT42). All other experiments, including GT126PI have greatly distorted the rainfall patterns in their predictions with lot of spread, whereas R5T126PI could reproduce most of the observed precipitation features, even on day-3 of forecast. In particular, the regional model captured the heavy rainfall in the northern parts of Bay of Bengal region with reasonable accuracy on all the three days.

\section{High-resolution physical initialization within the regional spectral model}

In short-range weather prediction over the monsoon domain, it is important to have the appropriate physics to handle the broad scale features of precipitation. Although the physics of the model includes a cumulus parameterization scheme capable of capturing the magnitude of condensation 

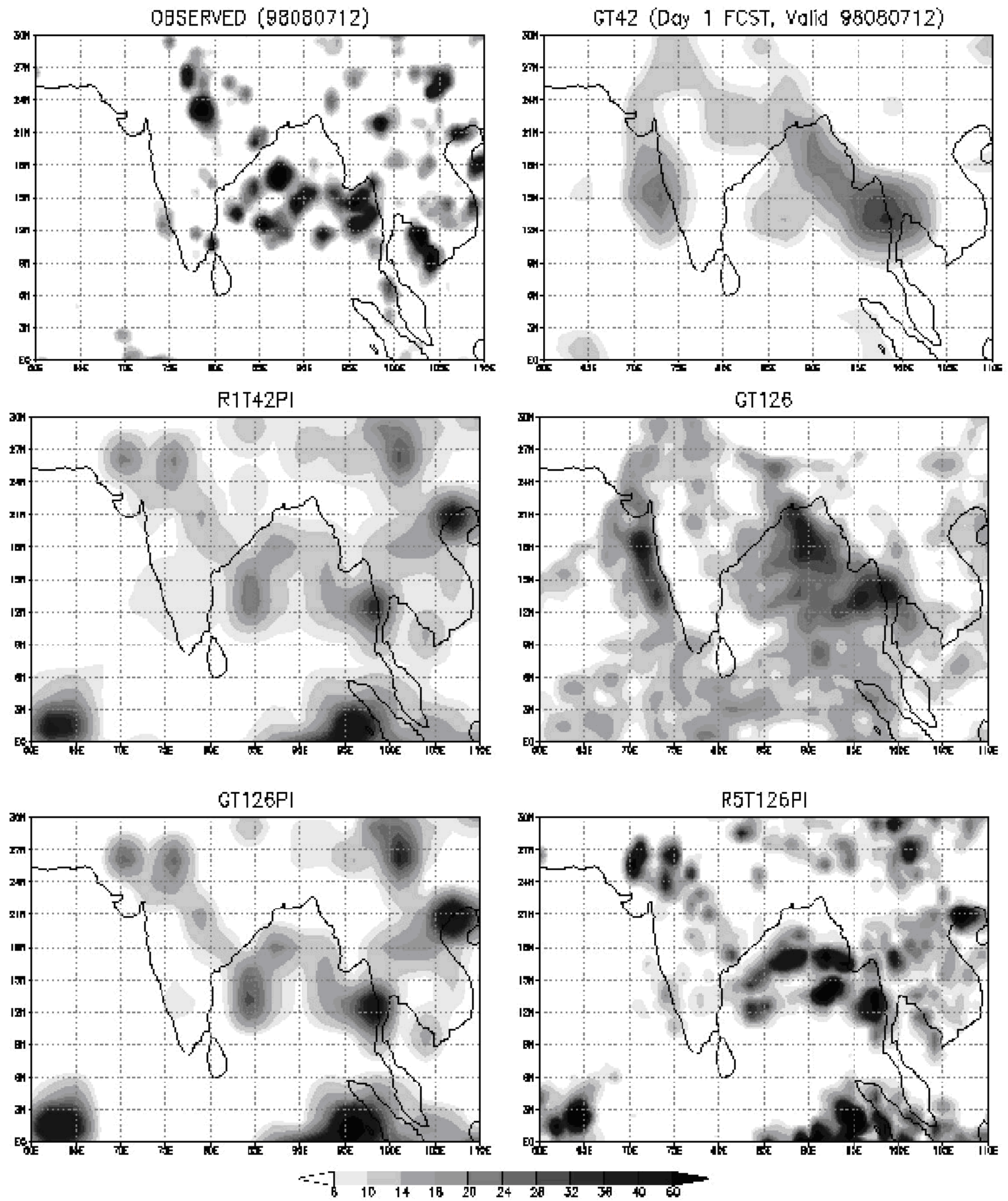

Figure 7(a). Day-1 precipitation forecast $\left(\mathrm{mm}_{\text {.day }}{ }^{-1}\right.$ ) valid on August 7, 1998. Observed precipitation is shown in the top left panel. (X-axis from $60^{\circ} \mathrm{E}$ to $110^{\circ} \mathrm{E}$, Y-axis from equator to $30^{\circ} \mathrm{N}$.) Details of experiments listed at the top of each panel are provided in table 2 .

heating, the forecast deteriorates rapidly, probably due to improper assimilation of rain rates. Physical initialization procedure assimilates observed rain rates in to the model atmosphere and improves the forecasting capabilities of the model (section 3). However, the high resolution of global 

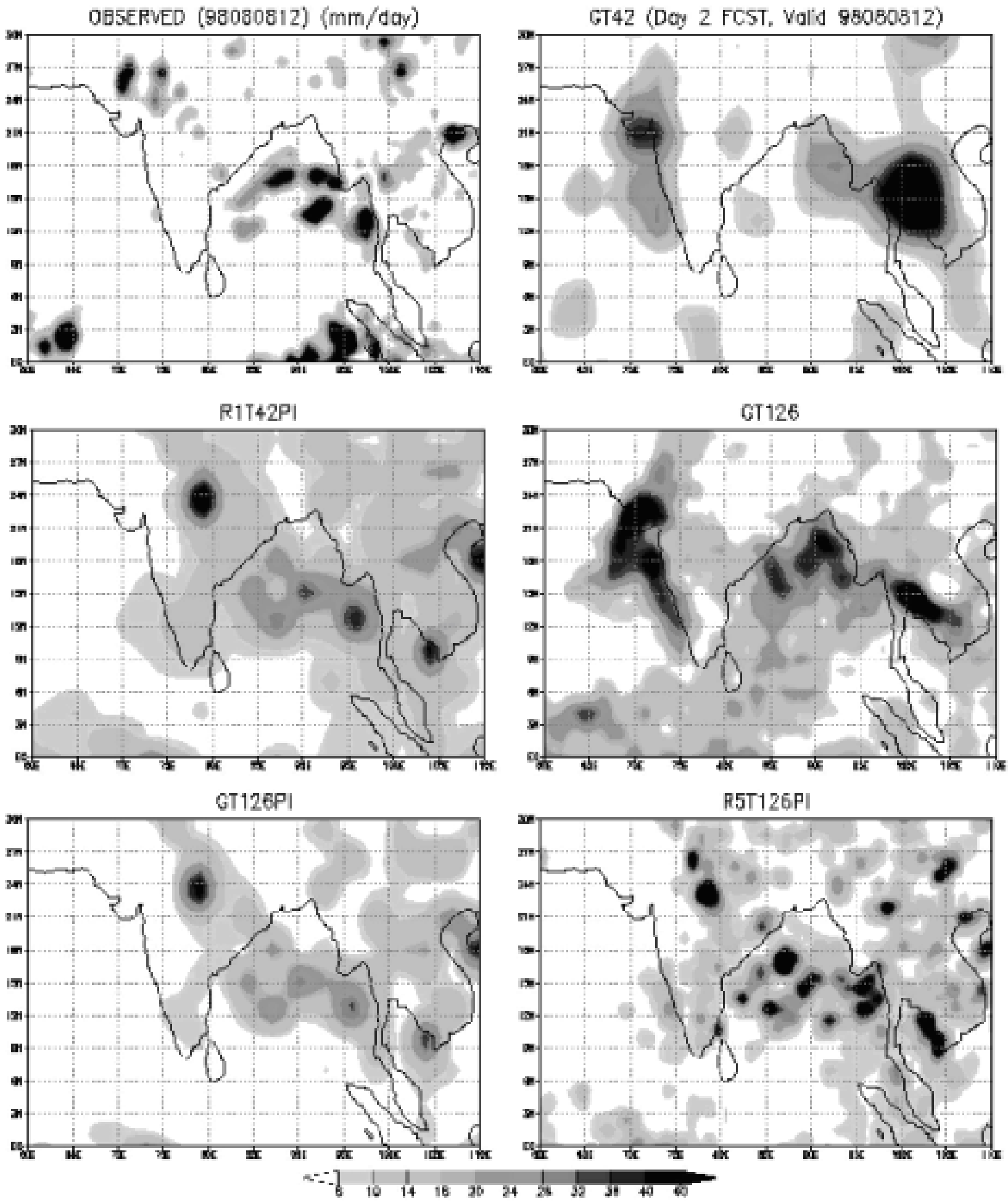

Figure 7(b). Same as figure 7(a) but for day-2 forecast valid on August 8, 1998.

model (T126) is still not sufficient to examine the meso-convective features of monsoon rainfall. The resolution of SSM/I microwave channels varies from roughly 30 to $60 \mathrm{~km}$. Physical initialization at this high resolution (about $50 \mathrm{~km}$ ) is required to nearly reproduce the 'observed' rain rates over the transform grid squares. The stronger divergence and heating fields evolve locally through this procedure, thereby improving both the precipitation forecasts and wind forecasts. Though R5T126PI 

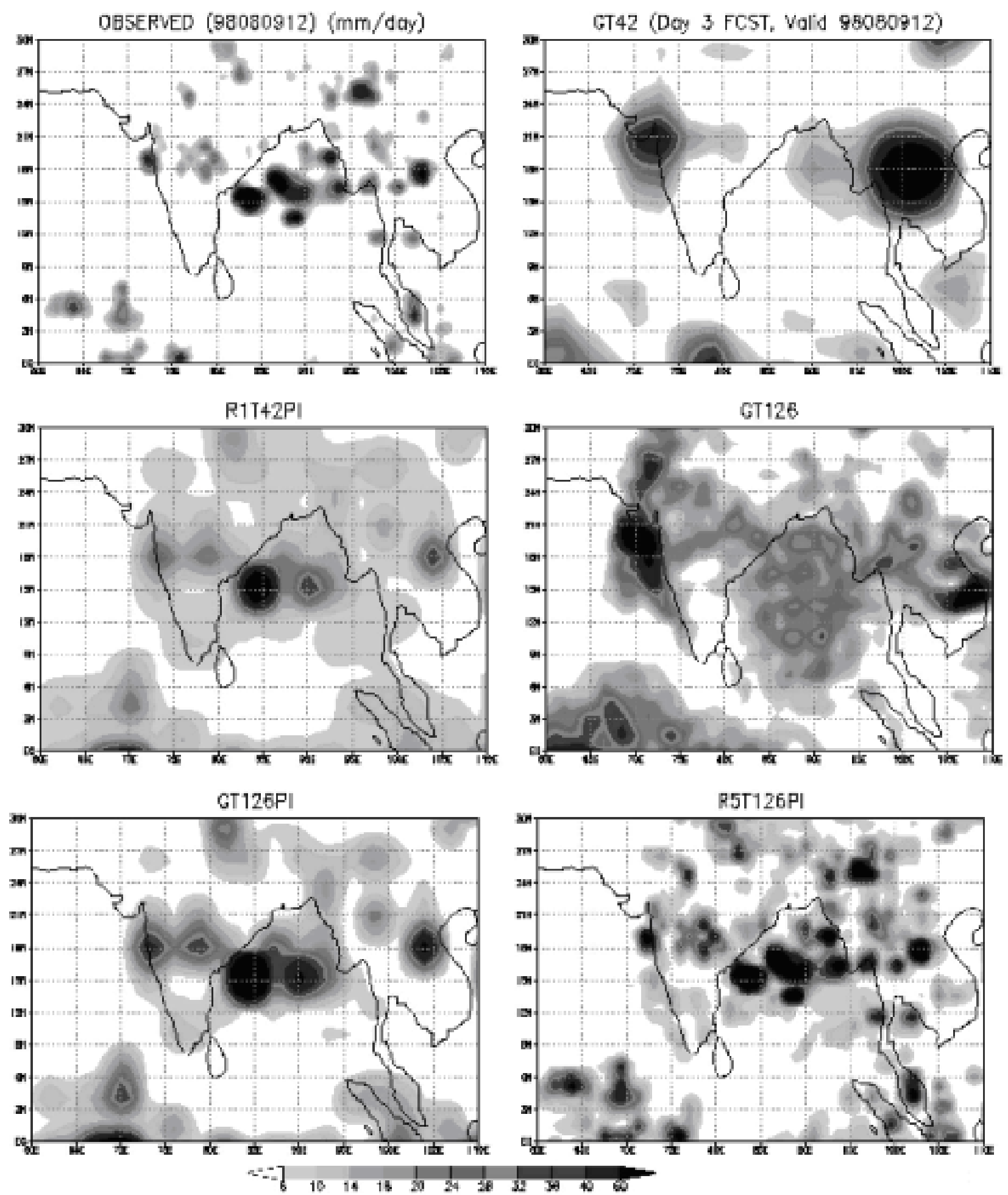

Figure 7(c). Same as figure 7(a) but for day-3 forecast valid on August 9, 1998.

takes into account the physically initialized base fields obtained from GT126PI, it still lacks some vital information required at its resolution. It is important to redesign the physical initialization procedure so that it can be applied directly at the regional model level to avoid loss of information through interpolation from global to regional grid. In a detailed study by Krishnamurti et al (1998), experiments carried out at the resolution of T255 (corresponding to about $50 \mathrm{~km}$ near the equator) were performed with a detailed physical initialization that described the initial distribution of 
convective elements quite reasonably. The computational difficulties in using a high resolution global model like T255 make it practically not viable for operational use. However, it is possible to improve the tropical precipitation forecasts up to 3 days by invoking physical initialization within a high resolution regional spectral model. In order to achieve this, temperature of the bottom slab of the atmosphere (soil temperature) is also prescribed during the physical initialization procedure. This was required since heat and moisture fluxes from the surface modify the state of the boundary layer, the mixing processes and the convective processes, which have relation to the overall circulation features. The ground flux (heat flux into the ground) is a small but very significant component of the surface energy budget. This flux is also related to the surface skin temperature. Since this flux is not measured directly, it needs to be parameterized for numerical models. The FSUGSM contains a PBL parameterization scheme based on the similarity theory (Businger et al 1971), which implicitly prescribes the ground temperature in calculating the surface fluxes. In order to improve this formulation, the soil temperature was prescribed into the model atmosphere based on the Force-Restore method (Stull 1988). The procedure for physical initialization, including the incorporation of soil temperature, at a high resolution, for the FSU modeling system is described below:

Step 1: Physical initialization of the global model with observed SSM/I and OLR merged rain rates and soil temperature during pre-integration phase. The ECMWF's observed (analyzed) soil temperature was also used along with the observed rain rates and OLR fields in this new physical initialization procedure.

Step 2: The global model fields were interpolated to the regional grid akin to the procedure explained in section 2.2 .

Step 3: The regional model was physically initialized (separately) using the observed SSM/I and OLR merged rain rates and soil temperature (interpolated to the regional model resolution) for that particular domain during the pre-integration phase. This creates the physically initialized fields for the regional model at a high resolution. The nudging coefficients used for the regional model were slightly different from the global physical initialization procedure. Here the relaxation coefficients were $6 \times 10^{-4} \mathrm{~s}^{-1}$ for vorticity and surface pressure, and $1 \times 10^{-4} \mathrm{~s}^{-1}$ for divergence. The increase in these values compared to the global model allows the iteration procedure to converge rapidly, approximately in 5-6 scans.

The global model and the regional model were then integrated for 72 hours starting with the
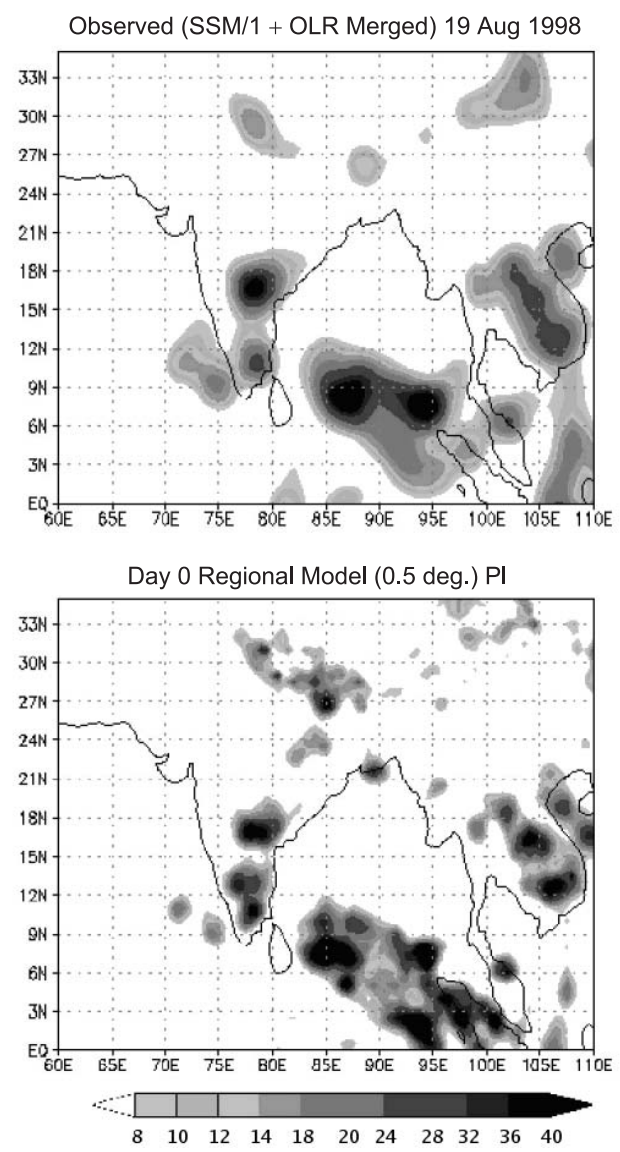

Figure 8. Day-0 regional precipitation fields $\left(\mathrm{mm}^{\mathrm{day}}{ }^{-1}\right)$ of FSUNRSM at 0.5 degree resolution, obtained through physical initialization at regional model level, valid on August 19, 1998. Top panel shows the observed estimates for the same day.

initial states obtained from the physical initialization procedure described above. Using this procedure, one set of experiments was performed with a start date of August 18, 1998. Figure 8 illustrates the initial rainfall from the regional physical initialization. The top panel shows the observed estimates of rainfall while the bottom panel shows the initialized rainfall estimates assimilated through physical initialization procedure applied separately for the regional model. A very high nowcasting skill of above 0.95 was realized through this procedure. This is much higher than the correlation between observed and initialized estimates of rain rates achieved by the global model through the same procedure. An example of a 3-day forecast of precipitation over the tropics from the regional model with physical initialization (Reg + PI + TSOIL) is presented in figure 9. A comparison of this forecast with satellite based estimates of precipitation (observed) provided in the extreme left panels and with other models, namely the control experiment (here GT126), and T126 with new 

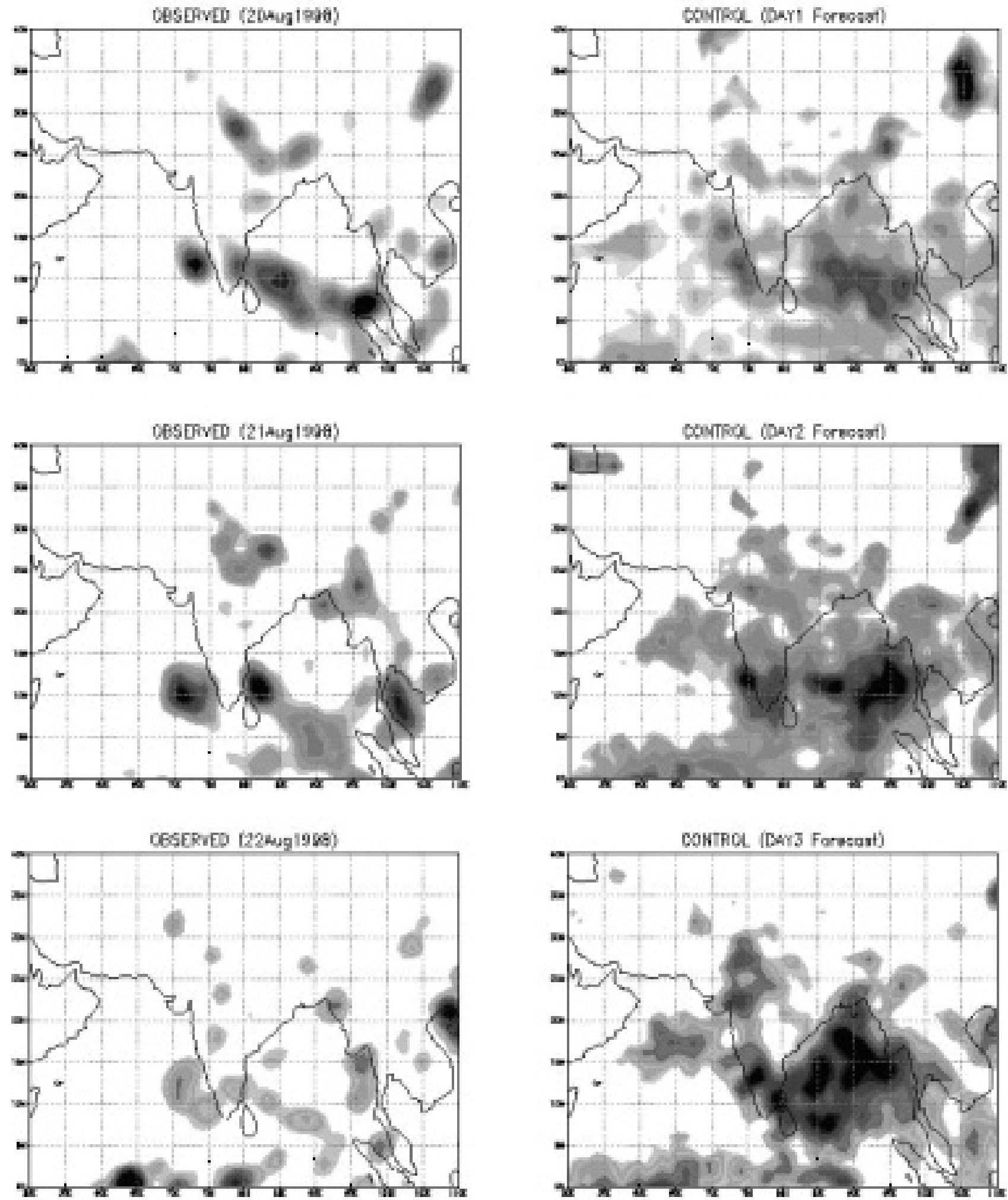

Figure 9. Day-1 to Day-3 forecasts of precipitation $\left(\right.$ mm.day $^{-1}$ ) valid on 20, 21 and 22, August 1998. PI+TSOIL represents the new physical initialization scheme that includes prescription of soil temperature. Observed precipitation is shown in the left panel valid for each day of forecast. Control is the G126 experiment. 

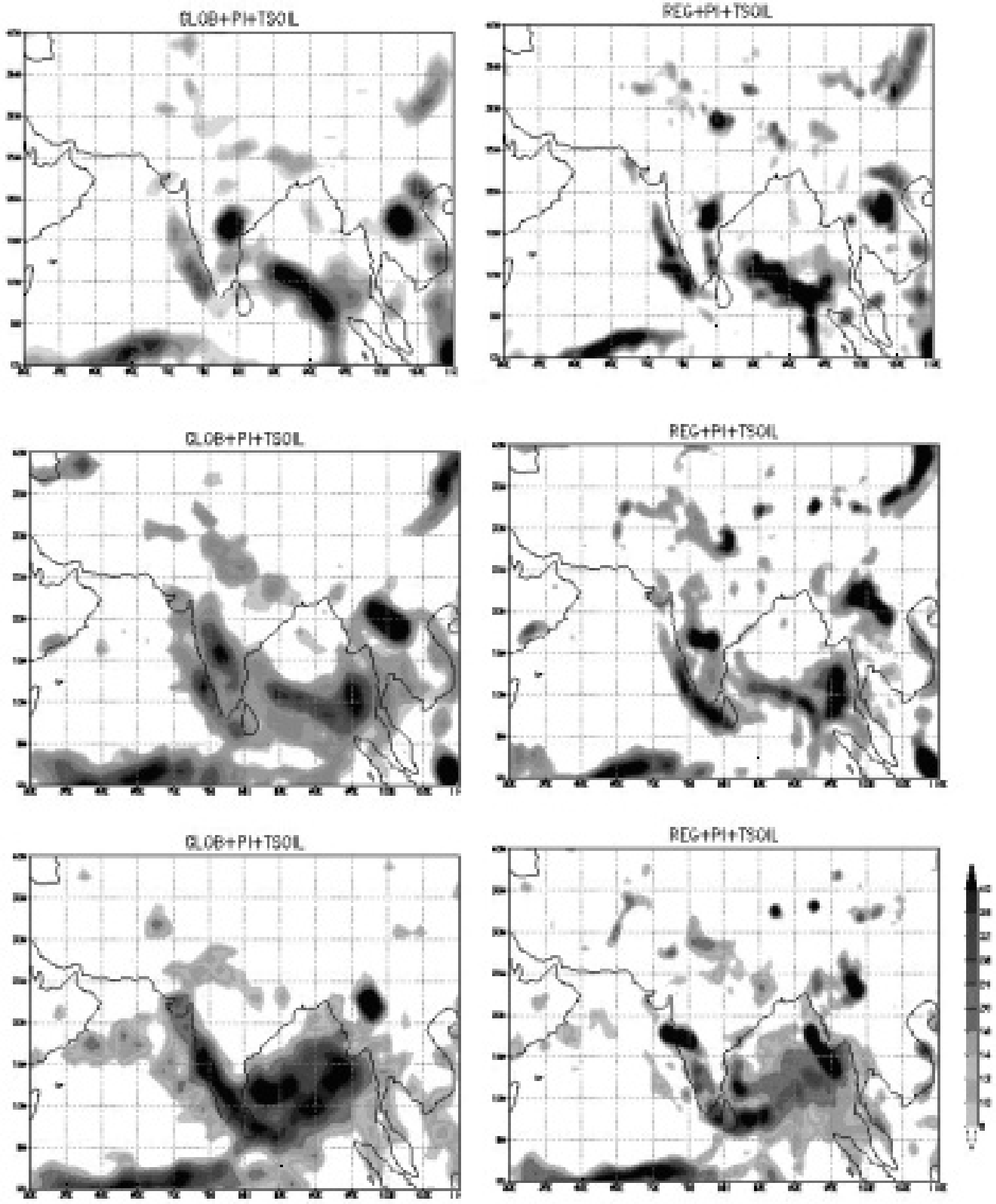

Figure 9. (Continued)

physical initialization (Glob + PI + TSOIL), indicates that this new experiment captures most of the precipitation features noted in the observed estimates on each day of the forecast. The impact of physical initialization on precipitation forecasts for the regional model is found to be much higher compared to that of the global model. Particularly, the rainfall that occurred over the southeast 


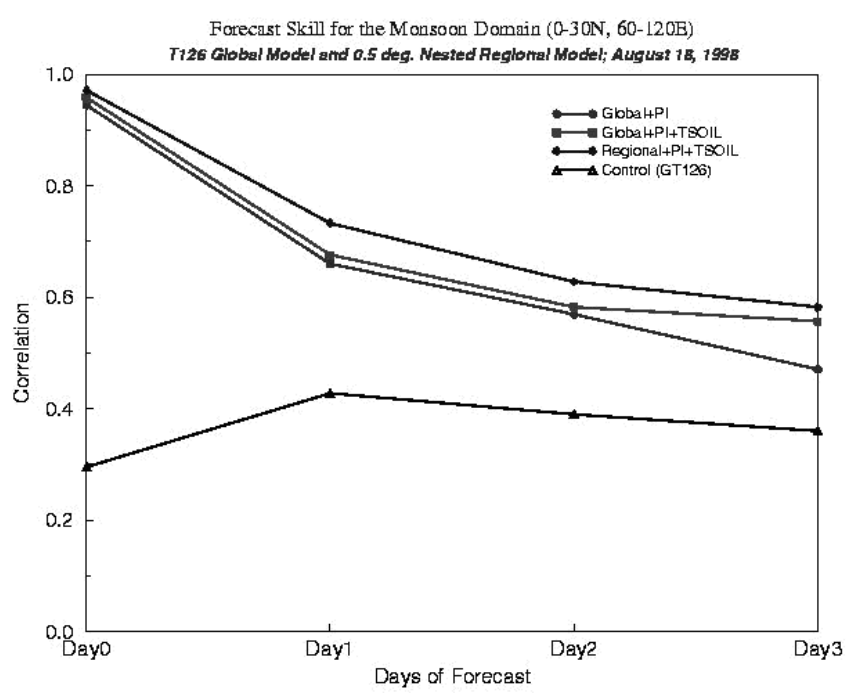

Figure 10. Correlation of precipitation estimates over the Indian monsoon domain for 3-day forecasts from (a) control (GT126), (b) GT126 with physical initialization (global+PI), (c) GT126 with new PI that assimilates soil temperature (global + PI + TSOIL) and (d) regional model with new PI that assimilates soil temperature (regional + PI + TSOIL).

Indian region and across the Western Ghats was reasonably captured by the regional model. Figure 10 illustrates the correlation of the observed and the 24-hourly rainfall totals $\left(\mathrm{mm}^{\left.- \text {day }^{-1}\right) \text { for }}\right.$ days 1, 2 and 3 of forecasts. It is apparent from these statistics that a much higher forecast skill of precipitation is realized from the use of physical initialization within the high resolution regional spectral model. Both the global models (GT126PI and GT126PI + TSOIL) exhibit a high correlation of about 0.6 for day-1 forecasts whereas a correlation of about 0.7 is obtained by the regional model with physical initialization that includes soil moisture initialization.

\section{Multimodel/multianalysis superensemble forecasts}

The process of integrating several model forecasts using statistical techniques (ensemble averaging) to reduce the forecast errors is found to be very useful, particularly in the context of shortto-medium range weather prediction. Since the improvement in model physics is inhibited by sparse observational network, many operational forecasting centers round the globe have started using ensemble forecasting methods to improve the skills of their forecasts. The success of superensemble methodology (Krishnamurti et al 1999, 2000a, 2000b, 2001) in providing superior weather and climate forecasts has opened gates for a new era of numerical weather forecasting. Unlike the

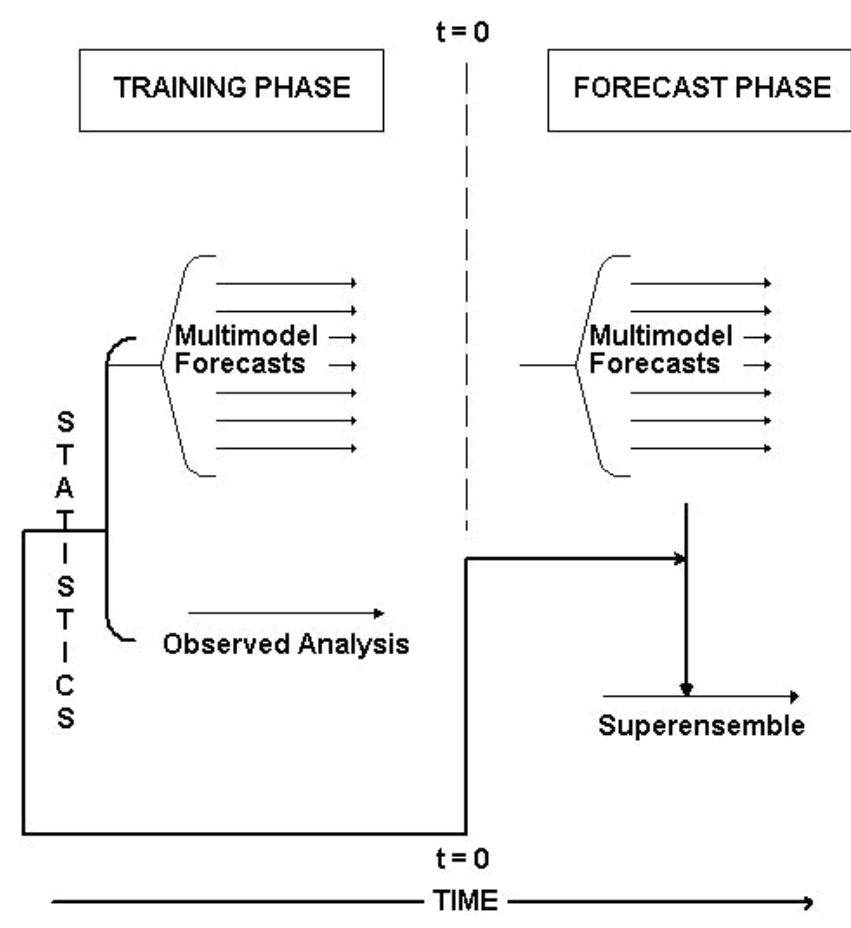

Figure 11. A schematic diagram of superensemble approach for multimodel forecasts.

ensemble methodology where different model forecasts are combined uniformly to minimize the forecast errors, superensemble removes the biases of its model members collectively at every grid point, for each variable at each level. Superensemble is developed by using a number of forecasts from a variety of NWP models. Along with the benchmark observed (analysis) fields, these forecasts are used to derive simple statistics on the past behavior of the models. These statistics, combined with multimodel forecasts, enable the construction of a superensemble. The skill of this multimodel statistical superensemble appears to far exceed those of the conventional ensemble averages and of the member models. The procedure used for designing the superensemble forecasts is outlined in figure 11. This defines a control (training) phase and a forecast phase. The multimodel forecasts are available during both phases. The observed fields (analysis) are available only for the control phase. A simple multiple linear regression of the model forecasts (anomaly fields) with respect to the observed fields, provide the useful statistics, which are deployed during the forecast phase. The creation of a multimodel superensemble prediction at a given grid point is described through the formula:

$$
S=\bar{O}+\sum_{i=1}^{n} a_{i}\left(F_{i}-\bar{F}_{i}\right)
$$

where $S$ is the superensemble prediction, $\bar{O}$ the time mean of 'observed' state, ' $a_{i}$ ' is weight for 


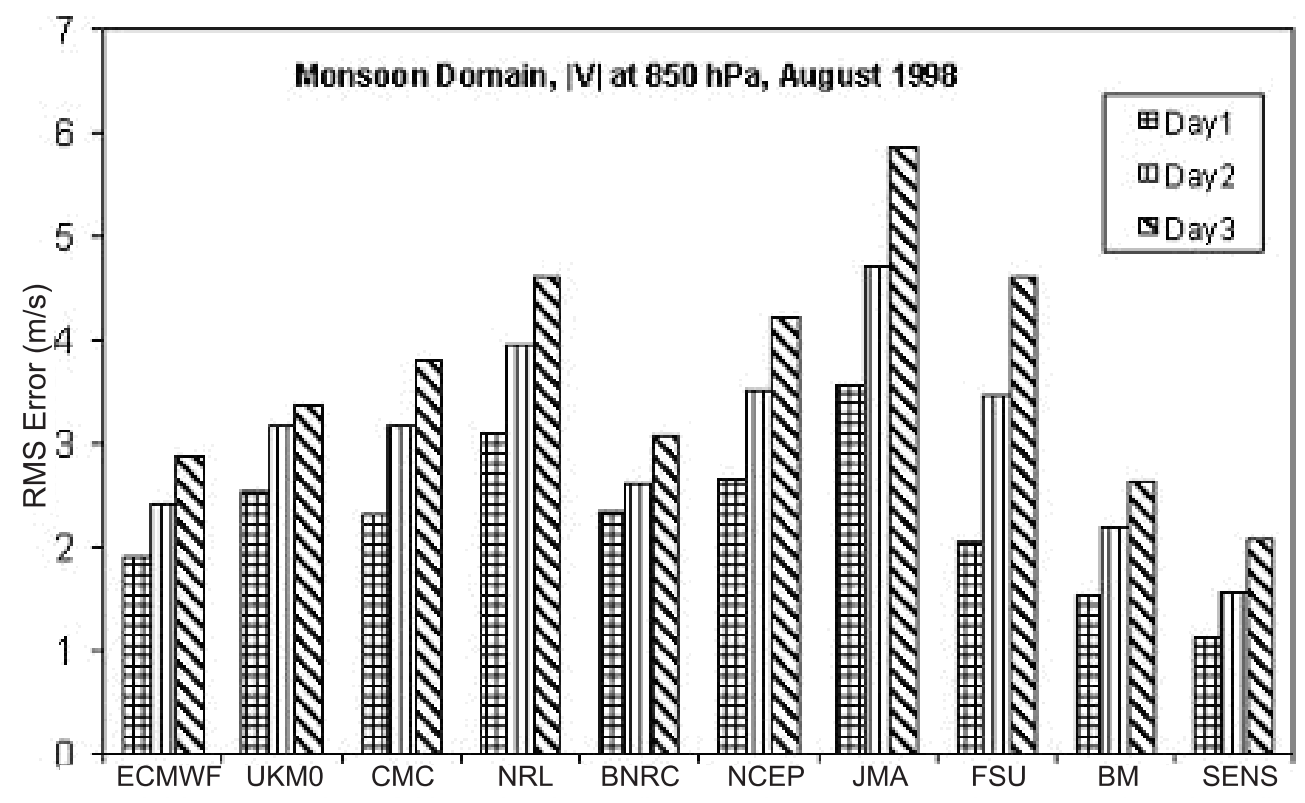

Figure 12. Mean RMS error of $850 \mathrm{hPa}$ total wind $|V|\left(\mathrm{ms}^{-1}\right)$ over the Indian domain August 1998, from multimodels, ensemble mean (EM) and superensemble (SENS), for day-1, day-2 and day-3 forecasts.

model ' $i$ ', $i$ is the model index, $N$ is the number of models used in constructing the superensemble, $\bar{F}_{i}$ is the time mean of prediction by model ' $i$ ' and $F_{i}$ is the actual prediction by model ' $i$ '. The weights ' $a_{i}$ ' are computed at each grid point by minimizing the function $G=\sum_{t}^{t \text {-train }}\left(S_{t}-O_{t}\right)^{2}$. Here ' $O$ ' is the observed state at time ' $t$ ' and ' $t$-train' is length of the training period.

\section{$5.1850 \mathrm{hPa}$ wind forecasts from multimodel superensemble}

In this study, seven NWP models were used to construct the superensemble forecasts: BMRC, ECMWF, UKMO, JMA, NRL, NCEP and CMC. These models have differing horizontal resolutions and a wide variety of physical parameterization schemes (Kumar 2000). In order to maintain uniformity, the model derived $850 \mathrm{hPa}$ wind forecasts were interpolated on to a 2.5 degree latitude/longitude grid. In total, the 7 models, each making 92 3-day forecasts with a start time at 12 UTC for each day starting from June 1, 1998 were used in constructing the superensemble forecasts. 61 days during June and July were treated as the control (training) period and the remaining 31 3-day forecasts for the month of August 1998 were used in the forecast phase. The $850 \mathrm{hPa}$ winds obtained from the experiments with FSUGSM and FSUNRSM during August 1998 were also used here for comparison with multimodels, ensemble mean and the superensemble.

The mean rms error statistics for total wind $\left(\mathrm{ms}^{-1}\right)$ over the Indian monsoon region at $850 \mathrm{hPa}$ for August 1998 corresponding to day-1, day-2 and day-3 of forecasts are shown in figure 12 . The superensemble was found to outperform various models by roughly $20 \%$ to $45 \%$ at each forecast range. A typical improvement of skill scores as high as $100 \%$ by the superensemble can be seen when compared to one of the poorer models (here JMA).

In figure 13, the streamlines and isotachs at $850 \mathrm{hPa}$ for a typical day-3 forecast valid on August 22, 1998 from the superensemble, best model (here ECMWF) and poor model (here JMA) are shown along with the observed (analysis) winds valid for that day. The improvements of the superensemble (shown in figure 13b) arise from a closer agreement of both wind direction and wind speed with respect to the analysis field over the monsoon domain. The southwesterlies were somewhat overestimated by the ECMWF forecast (figure 13c) and the overall patterns of wind circulation were displaced, whereas the superensemble could predict the observed features reasonably well. The forecasts from JMA (figure 13d) were totally distorted, both in terms of wind direction and speed. This kind of performance of superensemble on a day3 forecast can be considered as one of the major breakthroughs in the field of NWP.

\subsection{Rainfall forecasts from multianalysis superensemble}

The method of multianalysis superensemble for precipitation forecasts, originally developed by Krishnamurti et al $(1999,2001)$ utilizes a similar approach described in section 5.1. Here, 
(a) OESERVED (AUG.22,1998)

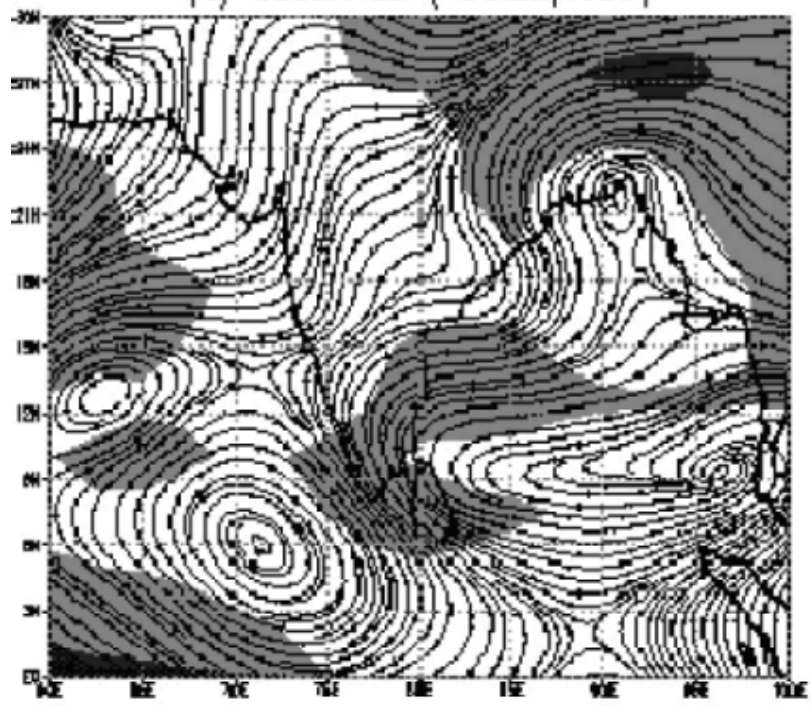

(b) SUPER ENSEMBLE (DAY3)

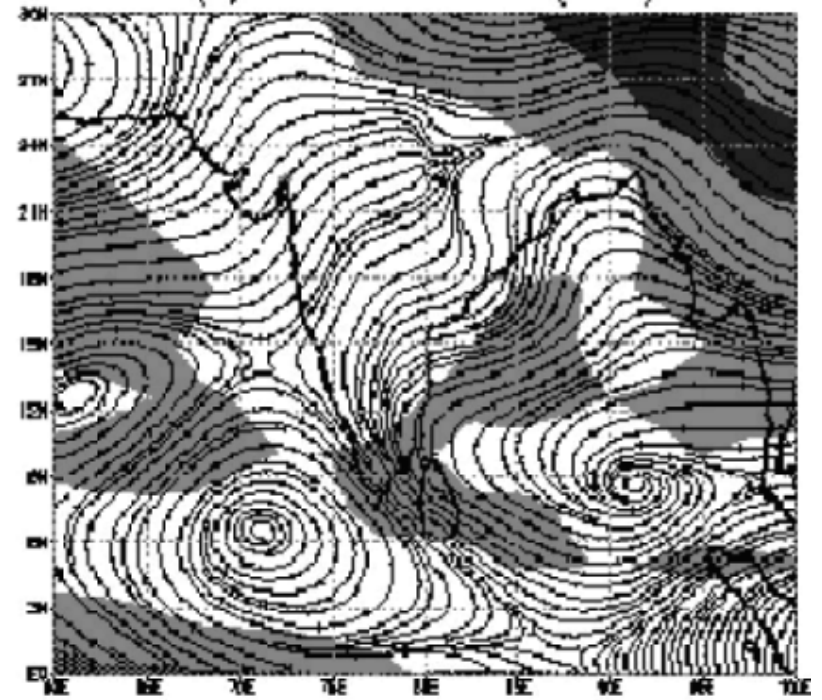

(d) POOR MODEL

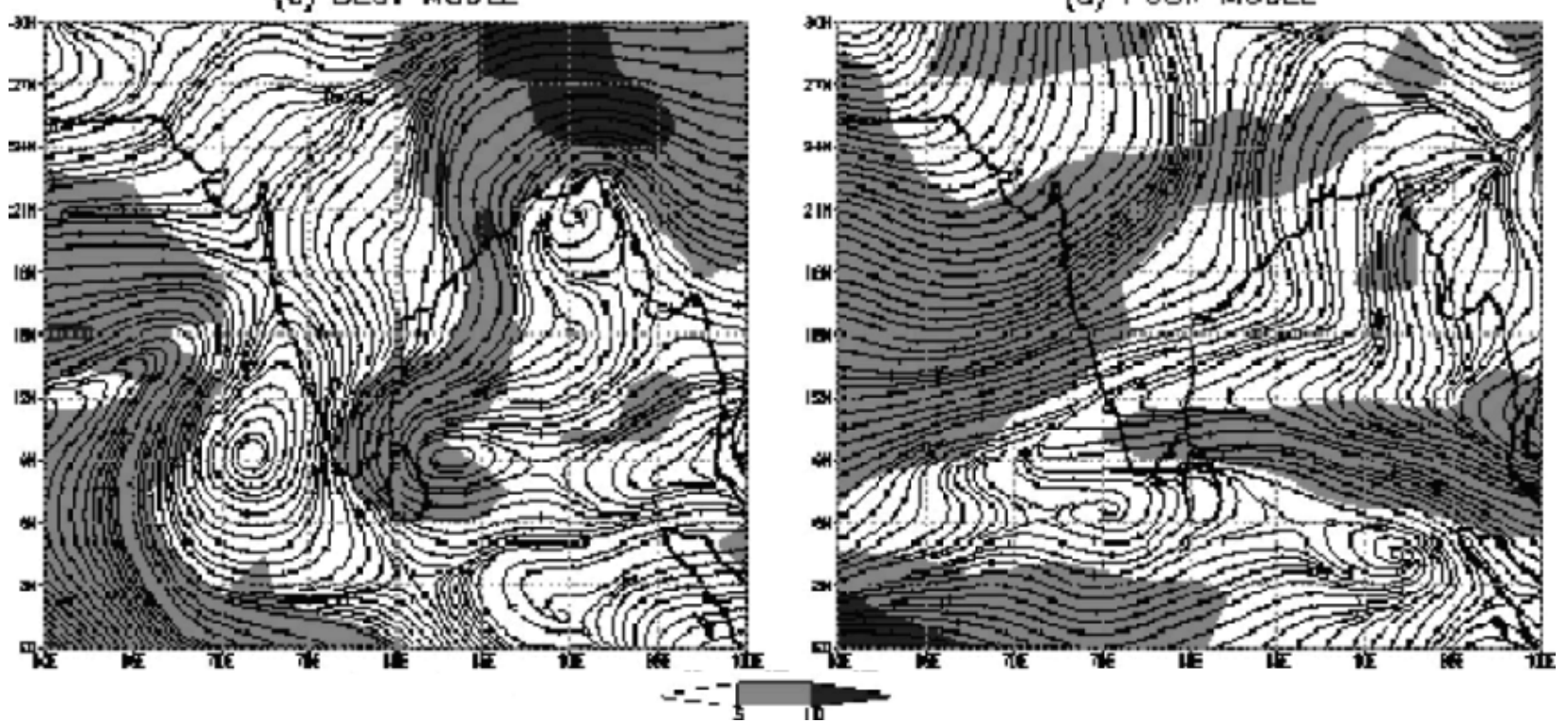

Figure 13. $850 \mathrm{hPa}$ streamlines and isotachs for day-3 forecast valid on August 22, 1998. Magnitude of wind (m s $\left.{ }^{-1}\right)$ is shaded. (a) Observed, (b) superensemble, (c) best model (here ECMWF) and (d) poor model (here JMA). (X-axis from $60^{\circ} \mathrm{E}$ to $110^{\circ} \mathrm{E}, \mathrm{Y}$-axis from $0^{\circ}-30^{\circ} \mathrm{N}$.)

combination of physical initialization based data assimilation of observed rainfall rates is used to generate a superensemble for precipitation forecasts. The process of physical initialization tends to have limitations beyond $24 \mathrm{~h}$ forecast, as it has been noted that the correlation of rain rates rapidly decreases from $24 \mathrm{~h}$ to $72 \mathrm{~h}$ of forecast. Inspired by the success of multimodel superensemble approach for NWP, the method of multianalysis superensemble was designed for use in precipitation forecasts over the tropics. This method is similar to the multimodel superensemble forecasting shown in figure 11, except that instead of multimodels, different versions of a single model (here the FSUGSM T126) were used with physical initialization of observed rain rates derived from different rain rate algorithms listed in table 3. Description of these algorithms is provided in Krishnamurti et al (2001) and Kumar (2000). The basic rain rate data sets were derived from the TRMM satellites and the SSM/I data from U.S. Air Force DMSP satellites. A total of 155 precipitation forecasts from 5 different versions of FSUGSM were conducted using these rain rate algorithms, of which 
Table 3. The rain rate algorithms used in the multianalysis superensemble.

\begin{tabular}{lll}
\hline Algorithm & \multicolumn{1}{c}{ Brief details } & \multicolumn{1}{c}{ Reference } \\
\hline Control & No physical initialization & Kummerow et al (1996) \\
GPROF & Uses SSM/I data & Olson et al (1990) \\
OLSON & Cal/Val Algorithm. Uses SSM/I data & Berg et al (1998) \\
\hline FERRARO & & Ferraro and Marks (1995) \\
$2 \mathrm{~A} 12$ & NOAA NESDIS Algorithm. Uses SSM/I data. & Kummerow et al (2000) \\
TMI 2A12 + SSM/I GPROF & Uses NASA TRMM TMI data & Kummerow et al (2000) \\
\hline
\end{tabular}

150 were used for the control (training) phase and 5 for the forecast phase. The control experiment did not include any physical initialization and had the lowest skill among all experiments. A relationship between the multianalysis forecasts and the best observed estimates of daily rain rate was determined through multiple regression based statistical weights that vary in space. During the forecast period, the statistics obtained in the control period were applied to the multianalysis forecasts to obtain the superensemble forecasts. The forecasted rain rates for day-1, day- 2 and day-3 were then compared with the multianalysis member model forecasts and best observed estimates. The multianalysis superensemble forecasts were found to be superior to that of the other models. These forecasts also demonstrated the large impact of TRMM data sets on prediction of tropical rainfall. Forecast skill from the proposed superensemble approach improved enormously when the TRMM/SSMI based rain rates were used as a benchmark for the definition of the superensemble statistics and for the forecast verification.

The mean RMS error of precipitation $\left(\mathrm{mm}\right.$ day $^{-1}$ ) on day-3 of the forecast (for the period from August 1 to 5, 1998) is shown in figure 14. The superensemble had minimum errors compared to other members of the multianalysis and also the FSUGSM and FSUNRSM experiments described in earlier sections. RMS error from the superensemble stayed close to about $5 \mathrm{~mm}$.day ${ }^{-1}$ while other members of the superensemble had errors ranging from 7 to $10 \mathrm{~mm}$.day ${ }^{-1}$. An example of a day-3 forecast of precipitation (mm.day $\left.{ }^{-1}\right)$ valid on August 4, 1998 is shown in figure 15. Here the top left panel shows the observed fields from TRMM-2A12 + DMSP-SSM/I data sets. Forecasts from different versions of the FSU model (listed in table 3) are displayed along with the superensemble (SUPER), FSUGSM (GT126PI) and FSUNRSM (R5T126PI). The best model among the multianalysis members here is the one that used TRMM + SSM/I data sets for physical initialization. Invariably, forecasts from the superensemble exhibited the highest skill here, having a close match with the observed patterns of rainfall over the Indian region. All other members had difficulties in carving out the precipitation patterns across this region, with a huge spread of heavy precipitation along the central and northern parts of India.

The results shown here are typical of superensemble, which are being noted in almost all the days of forecast. The major question that arises is how the superensemble pushes the skill to superior levels when the skill of the member models is much less. More than 40\% improvement simply comes from the relationship of the forecasts from multianalysis members forecasts and the observed fields during the training period. It was also noted that the superensemble performs better than simple ensemble mean of the forecasts, Krishnamurti et al (1999). The superensemble procedure evidently removes the local rainfall bias of the forecasts arising from the use of different rain rate algorithms. The collective bias removal gives superior results compared to the removal of the bias of the individual members (and averaging such results). The feasibility of real time precipitation forecasts using a multianalysis superensemble has emerged from these experiments, and at present, real time 5-day global precipitation forecasts are produced on a daily basis at FSU and are posted on the website (http://lexxy.met.fsu.edu/rtnwp).

\section{Summary and conclusions}

The primary goal of this study was to improve the skills of the numerical weather prediction over the Indian subcontinent. This has been accomplished through the use of a high-resolution nested regional spectral model with physical initialization. Current computing resources tend to limit the resolution of global models, justifying the use of high-resolution regional models in studying complex weather patterns like monsoon and tropical storms. The nested regional spectral model developed by Cocke (1998) was used in this study to assess the impact of resolution and physical initialization of observed 


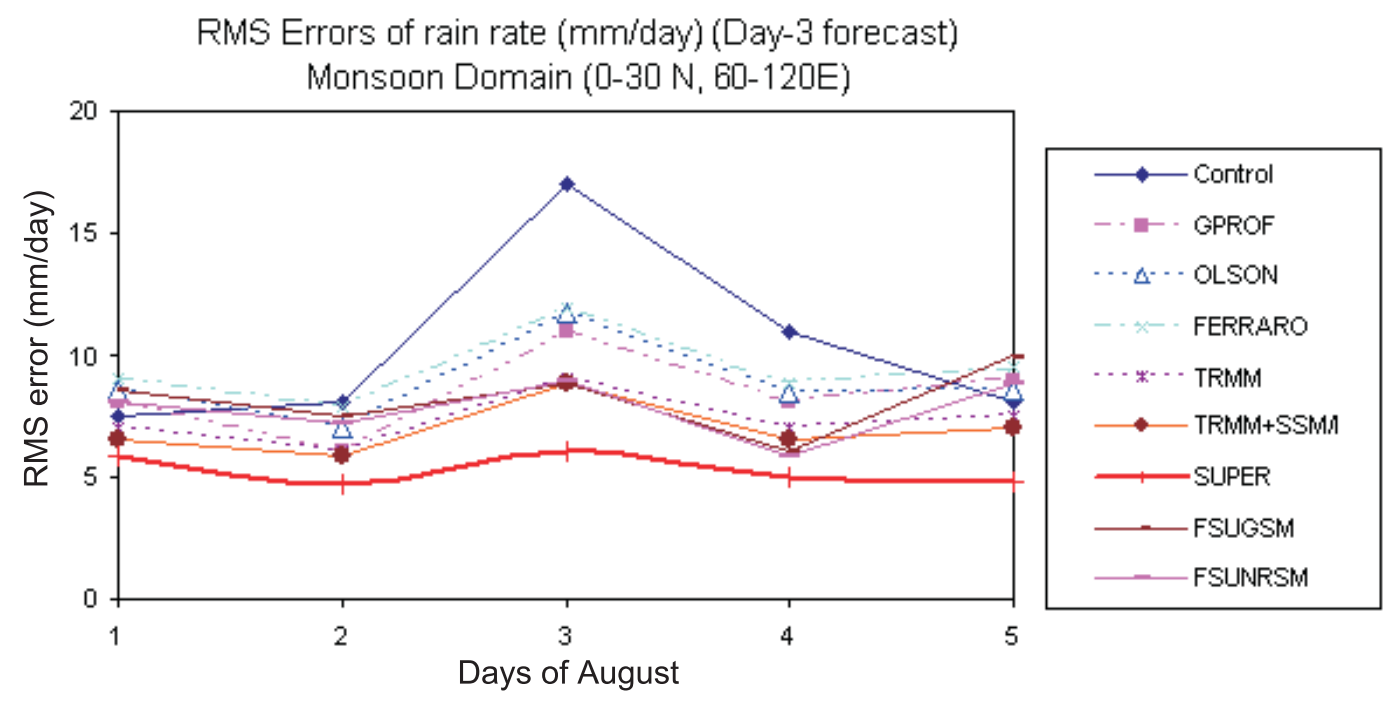

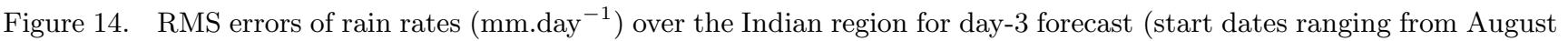
1 to 5,1998 ). Control is GT126. GPROF, Olson, Ferraro, TRMM and TRMM + SSM/I are multianalysis components of FSUGSM with PI.

rain rates on short-range weather prediction over the Indian subcontinent. The compatibility of this regional spectral model with the FSU global spectral model makes it more convenient for operational real time forecast initiative. The forecast skills of the regional spectral model with physical initialization of observed rain rates during the preintegration phase were superior to the skills of both the low-resolution global/regional models and the control experiments without physical initialization. The regional spectral model at 0.5 degree resolution has shown a higher skill for short-range (up to 3 days) forecasts during the monsoon season over the Indian subcontinent. The wind forecasts and precipitation rates predicted by the regional model were found to be more accurate and had a closer match to the observed fields.

From all the experiments described in this study, FSUNRSM was found to provide vast improvement over the FSUGSM. The greatest increase in performance occurred during day- 1 forecast and decreased rapidly by the day- 3 forecast. Improvement of the skills beyond day- 1 forecast were found possible through the use of physical initialization where observed measures of rain rates obtained from satellite data sets were assimilated directly at the regional spectral model level in the pre-integration phase, along with assimilation of soil temperature analysis. A high correlation of 0.95 was achieved for nowcasting of precipitation through this procedure. The correlation of rain rates for days 1, 2 and 3 of forecasts were found to be of the order of $0.7,0.6$ and 0.55 respectively and these correlations were much higher compared to experiments from all other versions of the FSU spectral model. Physical initialization appears to be a powerful tool for short-range forecasting of tropical precipitation. This procedure enhances the definition of mesoscale divergence, vorticity, vertical motion, convective heating and the surface pressure tendencies (Krishnamurti et al 1991), thereby increasing the forecast skill of tropical precipitation. Physical initialization also provides consistency among the model precipitation, evaporation, convergence of moisture flux and local changes in the precipitable water through a set of reverse physical algorithms and Newtonian relaxation (nudging) procedure. FSU model has a general tendency of overestimation of precipitation (a typical nature of modified Kuo scheme for cumulus parameterization). Though the 'bull's eye' pattern is noticed in the results, it is not certain at this time to attribute this to PI procedure alone. More careful evaluation of PI will be presented in a future publication where we used T255L29 version of FSU model and TRMM rain rates for PI.

The notion of superensemble, whose skills appear to far exceed those of conventional ensemble mean and member models, is based on the collective bias removal of member models at every grid location for each variable. Seven different operational global models were chosen in this study to construct the multimodel superensemble that includes a training and a forecast phase. The 3-day forecasts of $850 \mathrm{hPa}$ zonal and meridional winds for the months of June, July and August 1998 from all member models were used along with the observed (analysis) fields from ECMWF. The 61-day data from June and July were used in the training (control) phase to derive independent 

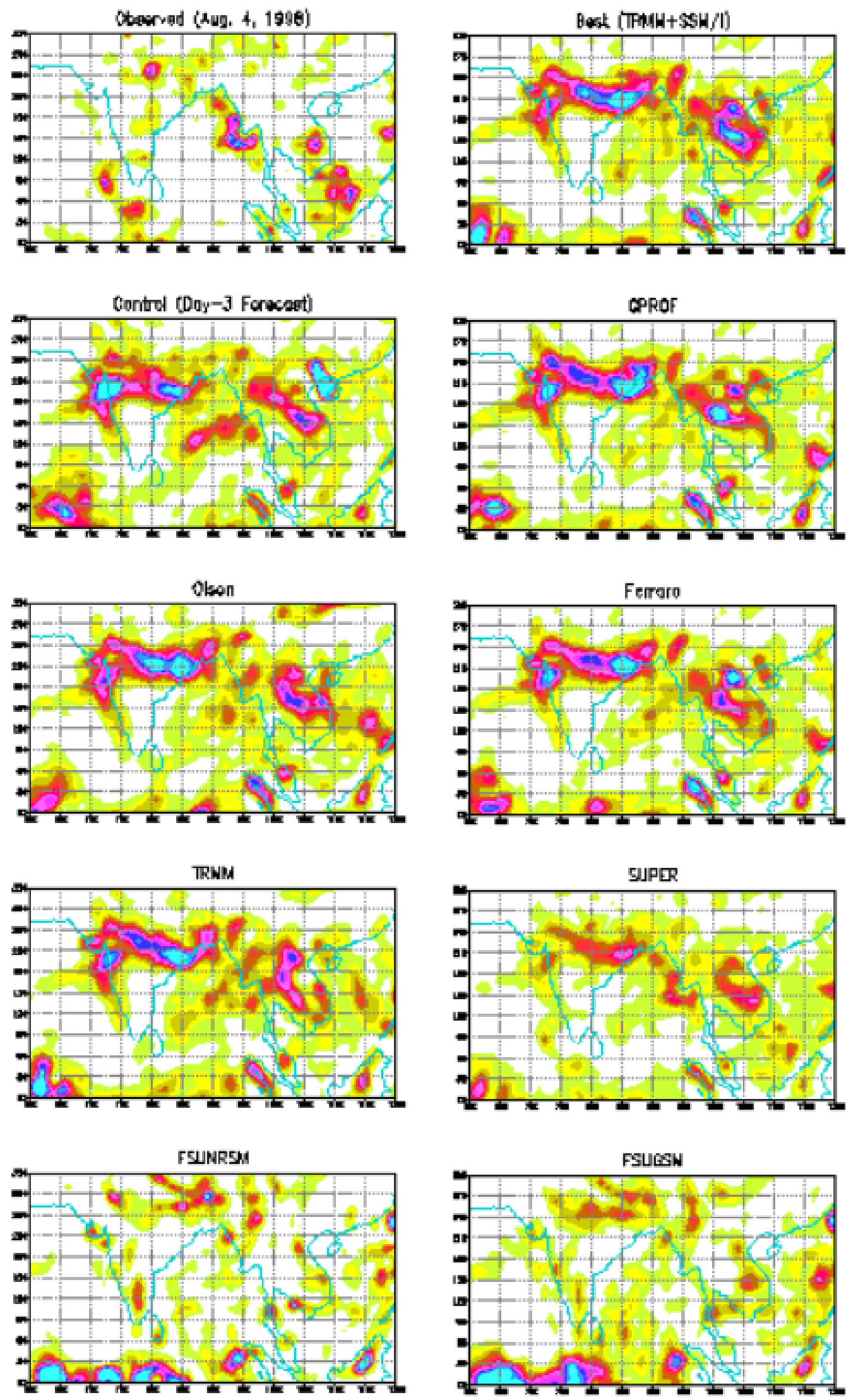

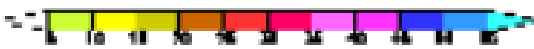

Figure 15. An example of day-3 forecast of precipitation $\left(\mathrm{mm}^{-\mathrm{day}}{ }^{-1}\right.$ ) valid on August 4, 1998 for the Indian monsoon region. Observed estimates shown in the upper left panel are from TRMM and SSM/I. Different panels show forecasts from multianalysis members, superensemble, FSUGSM and FSUNRSM. (X-axis from $60^{\circ} \mathrm{E}$ to $120^{\circ} \mathrm{E}$, Y-axis from $0^{\circ}-30^{\circ} \mathrm{N}$.) 
Table 4. Correlation of precipitation forecasts from different experiments.

\begin{tabular}{lcccc}
\hline Forecast experiment & Day 0 & Day 1 & Day 2 & Day 3 \\
\hline Without physical initialization (Control - GT126) & 0.35 & 0.40 & 0.35 & 0.30 \\
With physical initialization (Global Model - GT126PI) & 0.87 & 0.58 & 0.42 & 0.35 \\
With high-resolution physical initialization & 0.95 & 0.71 & 0.62 & 0.55 \\
$\quad$ Regional Model - PIR5T126PI) & 0.93 & 0.73 & 0.65 & 0.58 \\
Multianalysis superensemble & & & \\
\hline
\end{tabular}

sets of regression coefficients for all grid points at each forecast range. Using these coefficients in the forecast phase (here August 1998), this new approach succeeded in achieving remarkable improvements in the forecasting skills. The multimodel superensemble method greatly enhanced the skills of wind forecasts at $850 \mathrm{hPa}$ over the monsoon domain and it outperformed all other member models, including the conventional ensemble mean where all member models are given uniform weight irrespective of their past performance. RMS errors of superensemble forecasts for $850 \mathrm{hPa}$ winds over the Indian region were reduced by about $20 \%$ compared to the best model. The multianalysis superensemble method provided a new approach to produce more reliable rainfall forecasts over the Indian subcontinent. Each of the multianalysis components come from the use of the FSU global spectral model physically initialized using rain rate estimates retrieved from different algorithms for the TRMM/TMI and SSM/I data sets. A major improvement in the skills of rainfall forecasts is obtained through this method. The essential nature of precipitation over the tropics was meso-convective in character, and the loss of accuracy of the larger scale models was sufficient to offset any positive impacts from simple changes in the initialization procedures. The multianalysis superensemble proposed here looks at past rainfall observations derived from the TRMM and the DMSP satellites' microwave instruments. Inclusion of the detailed past rainfall data sets is a unique feature of this superensemble. The forecasts from the multianalysis data project the past relationship (between model forecasts and the observed rain) into the future, thereby providing a very high skill for short-range rainfall forecasts.

The results presented in this paper mainly deal with five types of experiments based on

(a) global model (and its resolution);

(b) physical initialization for the global model (combined with varying resolution);

(c) regional model (based on physically initialized global model data sets);

(d) physical initialization for the regional model; and

(e) superensemble.
The skills of the short-range forecasts increase from (a) to (e). This is summarized through the correlation of the observed and predicted rainfall skills over the Indian monsoon domain from day1 through day-3 from different experiments conducted in this study (table 4 ). The results obtained from these different experiments provided a great insight into the numerical weather prediction capabilities over the Indian subcontinent. The highresolution regional model with physical initialization has shown promising results in this endeavor. More experiments are needed with this regional model to further enhance its forecasting capabilities. The prescription of soil moisture through force-restore method needs better formulation and presently some work is going on in this direction. The multimodel/multianalysis superensemble approach is also another area where vast improvements are taking place. The Guass-Jordan method of elimination applied in the construction of covariance matrices in the multiple linear regression procedure is found to be sensitive to the singular values. Different methods like Singular Value Decomposition (SVD), Kalman Filter, EOF, an area of future work would be to explore the usefulness of Singular Value Decomposition (SVD), Z-transforms, Empirical Orthogonal Functions (EOFs) and cyclostationary EOFs are being used to remove the ill conditioning of the covariance matrices (Yun et al 2003). Many other modifications like optimizing the number of training days and the number of multimodels required in the training phase of the superensemble are also being carried out at present and the performance of superensemble is being tested for tropical cyclone forecasts (Kumar et al 2002; Williford et al 2002) and flood forecasts apart from seasonal climate forecasts and real-time numerical weather prediction.

\section{Acknowledgements}

The research reported here was funded by NASA grant Nos. NAG5-9662 and NAG8-1537; NSF grant Nos. ATM-9910526 and ATM-0108741 and FSURF COE. We acknowledge the constructive comments of the reviewers and from the editor in improving the quality of this manuscript. 


\section{Appendix 1: The Florida State University Global Spectral Model (FSUGSM)}

- Independent variables: $\lambda, \theta, \sigma, t$.

- Dependent variables: vorticity, divergence, temperature and moisture variable (dew point depression).

- Model variables are staggered in the vertical using Charney-Phillips vertical discretization vorticity, divergence, wind and geopotential are located at layer interface while temperatures, specific humidity and vertical velocity are assigned at the center of the layer. The vertical grid has higher resolution in stratosphere and in planetary boundary layer.

- Time integration scheme: the divergence equation, thermodynamic equation and pressure tendency equation are integrated implicitly while for vorticity equation and moisture continuity equation explicit time integration scheme is used. The tendencies of the physical processes are integrated using a forward time integration scheme.

- Space differing scheme: Spectral in the horizontal; centered differences in the vertical for all variables except moisture which is handled by an upstream differencing scheme.

- Surface topography is based on envelope orography (Wallace et al 1983).

- Parameterization of physical processes:

- Deep convection: based on modified Kuo cumulus parameterization scheme (Krishnamurti et al 1983; Krishnamurti and Bedi 1988; Kuo 1965, 1974), where the moistening and mesoscale convergence parameters are obtained from $700 \mathrm{hPa}$ vorticity and mean vertical velocity averaged over cloud depth through a regression relation.

- Shallow convection (Tiedke 1984).

- Dry convective adjustment.

- Large-scale condensation (Kanamitsu 1975). The scheme accounts for evaporation of falling precipitation.

- Surface fluxes of heat, moisture and momentum are calculated using similarity theory (Businger et al 1971). For low speeds $\left(\leq 5 \mathrm{~m} \mathrm{~s}^{-1}\right)$ moisture fluxes are calculated following Beljaars and Miller (1990).

- Vertical distribution of fluxes in the free atmosphere is based on stability (Richardson number) dependent exchange coefficient (Louis 1979).

- 4th order horizontal diffusion (Kanamitsu et al 1983).

- Vertical diffusion based on the K-theory for representation of energy sinks in the model (Louis 1979).
- Long and shortwave radiative fluxes based on a band model (Harshvardan and Corsetti 1984; Lacis and Hansen 1974).

- Diurnal solar cycle.

- Parameterization of low, medium and high clouds for radiative transfer calculation is based on threshold relative humidity. Fraction area of various cloud distribution configurations in the vertical is based on random overlap consideration.

- Surface temperatures: Prescribed over the oceans, while over the land a surface energy balance coupled to the similarity theory determines the surface temperature including its diurnal cycle (Krishnamurti et al 1991).

- Initialization: The initialization of the model is achieved in two stages:

- Nonlinear normal mode initialization (Kitade 1983), wherein the tendencies of first 5 modes with phase speed exceeding about $30 \mathrm{~m} \mathrm{~s}^{-1}$ are damped during the initialization. The slow moving higher modes are allowed to adjust freely.

- Physical initialization wherein moisture field, heat sources and sinks and divergence fields are initialized consistent with observed OLR and rain rates (Krishnamurti et al 1991).

Further details of the model including its mathematical formulation, description of the physical parameterizations and their application in FSUGSM are provided in detail in Kumar (2000).

\section{References}

Asselin R A 1972 Frequency filter for time integrations; Mon. Wea. Rev. 100 487-490.

Baer F 2000 Numerical weather prediction; Advances in Computers 52 91-157.

Beljaars A and Miller M 1990 A note concerning the evaporation from the tropical oceans: sensitivity of the ECMWF model to transfer coefficient of moisture at low wind speed; ECMWF Research Department Technical Memo 170, ECMWF, Reading, pp. 19.

Berg W, Olson W, Ferrao R, Goodman S J and Lafontaine F L 1998 An assessment of the firstand second-generation navy operational precipitation retrieval algorithms; J. Atmos. Sci. 55 1558-1575.

Businger J A, Wyngard J C, Izumi Y and Bradley E F 1971 Flux profile relationship in the atmospheric surface layer; J. Atmos. Sci. 28 181-189.

Cocke S 1998 Case Study of Erin using the FSU Nested Regional Spectral Model; Mon. Wea. Rev. 126 1337-1346.

Daley R C, Girard C, Henderson J and Simmonds I 1976 Short-term forecasting with a multi-level spectral primitive equation model. Part I: Model formulation; Atmosphere 14 98-116.

Epstein E S 1969 Stochastic-dynamic prediction; Tellus 21 $739-759$. 
Ferraro R R and Marks G F 1995 The development of SSM/I rain-rate retrieval algorithms using ground-based radar measurements; J. Atmos. Oceanic Technol. 12 755-770.

Harshvardan and Corsetti T G 1984 Long-wave parameterization for the UCLA/GLAS GCM; NASA Tech. Memo. 86072, Goddard Space Flight Center, Greenbelt, MD 20771, $52 \mathrm{pp}$.

Hoyer J M 1987 The ECMWF spectral limited area model; Proc. ECMWF Workshop on techniques for horizontal discretization in numerical weather prediction models, Shinfield Park, Reading, United Kingdom. ECMWF, pp. 343-359.

India Meteorological Department (IMD) 1999 Climate Diagnostics Bulletin 1998; IMD, New Delhi.

Juang H M and Kanamitsu M 1994 The NMC Nested Regional Spectral Model; Mon. Wea. Rev. 122 3-26.

Kalnay E 2002 Atmospheric Modeling, Data Assimilation and Predictability; Cambridge University Press, 512p.

Kanamitsu M 1975 On numerical prediction over a global tropical belt; Report No. 75-1, Department of Meteorology, Florida State University, Tallahassee, FL 32306, 142 pp.

Kanamitsu M, Tada K, Kuda K, Sato N and Isa S 1983 Description of the JMA operational spectral model; J. Meteor. Soc. Japan 61 812-828.

Kitade T 1983 Nonlinear normal mode initialization with physics; Mon. Wea. Rev. 111 2194-2213.

Krishnamurti T N, Low-Nam S and Pasch R 1983 Cumulus parameterization and rainfall rates II; Mon. Wea. Rev. $111815-828$

Krishnamurti T N, Ingles K, Cocke S, Pasch R and Kitade T 1984 Details of low latitude medium range numerical weather prediction using a global spectral model II: effect of orography and physical initialization; J. Meteor. Soc. Japan 62 613-649.

Krishnamurti T N and Bedi H S 1988 Cumulus parameterization and rainfall rates III; Mon. Wea. Rev. 116 583-599.

Krishnamurti $\mathrm{T}$ N, Xue J, Bedi $\mathrm{H}$ S, Ingles $\mathrm{K}$ and Oosterhof D 1991 Physical initialization for numerical weather prediction over the tropics; Tellus 43 53-81.

Krishnamurti T N 1995 Numerical Weather Prediction; Annu. Rev. Fluid Mech. 27 195-224.

Krishnamurti T N, Bedi H S and Han W 1998 Organization of convection and monsoon forecasting; Meteorol. Atmos. Phys. 65 171-181.

Krishnamurti T N, Kishtawal C M, LaRow T, Bachiochi D, Zhang Z, Williford C E, Gadgil S and Surendran S 1999 Improved skills for weather and seasonal climate forecasts from multi-model superensemble; Science $\mathbf{2 8 5}$ $1548-1551$

Krishnamurti T N, Kishtawal C M, LaRow T, Bachiochi D, Zhang Z, Williford C E, Gadgil S and Surendran S 2000a Multi-model superensemble forecasts for weather and seasonal climate; J. Climate 13 4196-4216.

Krishnamurti T N, Kishtawal C M, Shin D W and Williford C E 2000b Improving precipitation forecasts from a multi-analysis superensemble; J. Climate 13 42174227.

Krishnamurti T N, Surendran S, Shin D W, Correa-Torres R, Vijaya Kumar T S V, Williford C E, Kummerow C, Adler R F, Simpson J, Kakar R, Olson W and Turk F J 2001 Real Time Multianalysis/Multimodel Superensemble Forecasts of Precipitation using TRMM and SSM/I Products; Mon. Wea. Rev. 129 2861-2883.

Kumar T S V V 2000 High resolution short-range numerical weather prediction over the Indian subcontinent; Ph.D. Dissertation, Andhra University, Visakhapatnam, India, 295pp.
Kumar T S V V, Krishnamurti T N, Michael Fiorino and Nagata M 2002 Multimodel Superensemble Forecasting of Tropical Cyclones in the Pacific; Mon. Wea. Rev. 131 $574-583$.

Kummerow C, Olson W S and Giglio L 1996 A simplified scheme for obtaining precipitation and vertical hydrometeor profiles from passive microwave sensors; IEEE Trans. Geosci. Remote Sens. 34 1213-1232.

Kummerow C, Simpson J, Thiele O, Barnes W, Chang A T C, Stocker E, Adler R F, Hou, Kakar R, Krishnamurti $\mathrm{T}$ N, Wentz F, Ashcroft P, Kozu T, Hong Y, Iguchi T, Im E, Haddad Z, Huffman G, Ferrier B, Olson W S, Zipser E, Smith E A 2000 The status of the Tropical Rainfall Measuring Mission (TRMM) after two years in orbit; J. Appl. Meteor. 39 1965-1982.

Kuo H L 1965 On formation and intensification of tropical cyclones through latent heat release by cumulus convection; J. Atmos. Sci. 22 40-63.

Kuo H L 1974 Further studies of the parameterization of the influence of cumulus convection on the large-scale flow; J. Atmos. Sci. 31 1232-1240.

Lacis A A and Hansen J E 1974 A parameterization of the absorption of solar radiation in the earth's atmosphere; J. Atmos. Sci. 31 118-133.

Leith C E 1974 Theoretical skill of Monte Carlo forecasts; Mon. Wea. Rev. 102 409-418.

Lorenz E N 1963 Deterministic non-periodic flow; J. Atmos. Sci. 20 130-141.

Lorenz E N 1965 A study of predictability of a 28-variable atmospheric model; Tellus 17 321-333.

Lorenz E N 1968 The predictability of a flow which possesses many scales of motion; Tellus 21 289-307.

Louis J F 1979 A parametric model of vertical eddy fluxes in the atmosphere; Boundary Layer Meteorology $\mathbf{1 7}$ 187-202.

Olson W S, Fontaine F J, Smith W L and Achtor T H 1990 Recommended algorithms for the Retrieval of rainfall rates in the tropics using the SSM/I (DMSP-8). Manuscript. University of Wisconsin, Madison, 10pp.

Rajeevan M, Pai D S, Dikshit S K and Kelkar R R 2004 IMD's new operational models for long range forecast of south-west monsoon rain fall and their verification for 2003; Curr. Sci. 86 422-431.

Rama Rao Y V, Kar S C, Vijaya Kumar T S V, Kalsi S R, Hatwar H R and Roy Bhowmik S K 2005 Improvements in the weather prediction over the Indian region using Regional Spectral Model; Mausam 56 343-356.

Roache P J 1976 Computational Fluid Dynamics, Albuquerque: Hermosa, 446pp.

Roy Bhowmik S K and Prasad K 2001 Some characteristics of limited area model precipitation forecast of Indian monsoon and evaluation of associated flow features; Meteor. Atmos. Phys. 76 223-236.

Shin D W 2001 Short to Medium Range Superensemble Precipitation Forecasts using Satellite Products; Ph.D. Dissertation, Florida State University, Tallahassee, Florida, USA. 198pp.

Stull R B 1988 Boundary Layer Meteorology; Cambridge University Press, 355pp.

Tatsumi Y 1986 A spectral limited area model with time dependent lateral boundary conditions and its application to a multi-level primitive equation model; J. Meteor. Soc. Japan 64 637-663.

Thapliyal V and Kulshrestha S M 1992 Recent models for long range forecasting of southwest monsoon rainfall in India; Mausam 43 239-246.

Thapliyal V 1997 Preliminary and final long range forecast for seasonal monsoon rainfall over India; J. Arid Env. 36 $385-403$. 
Tiedtke M 1984 The sensitivity of the time-mean largescale flow to cumulus convection in the ECMWF model; Workshop on convection in large-scale numerical models, Reading, UK. ECMWF, 297-316.

Wallace J M, Tibaldi S and Simmons A J 1983 Reduction of systematic forecast errors in the ECMWF model through the introduction of envelope orography; Quart. J. Roy. Meteor. Soc. 109 683-718.

Williford C E, Correa-Torres R J and Krishnamurti T N 1998 Tropical Cyclone Forecasts Made with the FSU Global Spectral Model; Mon. Wea. Rev. 126 1332-1336.
Williford C E, Krishnamurti T N, Ricardo Correa Torres, Steven Cocke, Zaphiris Christidis and Vijaya Kumar T S V 2002 Real-Time Multimodel Superensemble Forecasts of Atlantic Tropical Systems of 1999; Mon. Wea. Rev. 131 1878-1894.

Yanai M, Esbensen S and Chu J H 1973 Determination of bulk properties of tropical cloud clusters from large-scale heat and moisture budgets; J. Atmos. Sci. 30 611-627.

Yun W T, Stefanova L and Krishnamurti T N 2003 Improvement of the multimodel superensemble technique for seasonal forecasts; J. Climate 16(22) 3834-3840. 